ARTICLE

\title{
p62/SQSTM1-droplet serves as a platform for autophagosome formation and anti-oxidative stress response
}

\author{
Shun Kageyama1,14, Sigurdur Runar Gudmundsson (10 2,14, Yu-Shin Sou ${ }^{3}$, Yoshinobu Ichimura1, Naoki Tamura ${ }^{4}$, \\ Saiko Kazuno (10 ${ }^{5}$, Takashi Ueno ${ }^{5}$, Yoshiki Miura ${ }^{5}$, Daisuke Noshiro ${ }^{6}{ }^{6}$, Manabu Abe ${ }^{7}$, Tsunehiro Mizushima ${ }^{8}$, \\ Nobuaki Miura (10 9 , Shujiro Okuda (1) 9 , Hozumi Motohashi (iD ${ }^{10}$, Jin-A Lee (1) ${ }^{11}$, Kenji Sakimura7, \\ Tomoyuki Ohe (1) ${ }^{12}$, Nobuo N. Noda (1) ${ }^{6}$, Satoshi Waguri (i) ${ }^{4}$, Eeva-Liisa Eskelinen (i) ${ }^{2,13 凶}$ \& \\ Masaaki Komatsu (iD ${ }^{1 凶}$
}

Autophagy contributes to the selective degradation of liquid droplets, including the PGranule, Ape1-complex and p62/SQSTM1-body, although the molecular mechanisms and physiological relevance of selective degradation remain unclear. In this report, we describe the properties of endogenous p62-bodies, the effect of autophagosome biogenesis on these bodies, and the in vivo significance of their turnover. p62-bodies are low-liquidity gels containing ubiquitin and core autophagy-related proteins. Multiple autophagosomes form on the p62-gels, and the interaction of autophagosome-localizing Atg8-proteins with p62 directs autophagosome formation toward the p62-gel. Keap1 also reversibly translocates to the p62gels in a p62-binding dependent fashion to activate the transcription factor Nrf2. Mice deficient for Atg8-interaction-dependent selective autophagy show that impaired turnover of p62-gels leads to Nrf2 hyperactivation in vivo. These results indicate that p62-gels are not simple substrates for autophagy but serve as platforms for both autophagosome formation and anti-oxidative stress.

\footnotetext{
${ }^{1}$ Department of Physiology, Juntendo University Graduate School of Medicine, Bunkyo-ku, Tokyo 113-8421, Japan. ${ }^{2}$ Molecular and Integrative Biosciences Research Programme, University of Helsinki, Helsinki 00014, Finland. ${ }^{3}$ Department of Cell Biology and Neuroscience, Juntendo University Graduate School of Medicine, Bunkyo-ku, Tokyo 113-8421, Japan. ${ }^{4}$ Department of Anatomy and Histology, Fukushima Medical University School of Medicine, Hikarigaoka, Fukushima 960-1295, Japan. ${ }^{5}$ Laboratory of Proteomics and Biomolecular Science, Research Support Center, Juntendo University Graduate School of Medicine, Bunkyo-ku, Tokyo 113-8421, Japan. ${ }^{6}$ Institute of Microbial Chemistry (BIKAKEN), Shinagawa-ku, Tokyo 141-0021, Japan. ${ }^{7}$ Department of Animal Model Development, Brain Research Institute, Niigata University, Niigata 951-8510, Japan. ${ }^{8}$ Picobiology Institute, Graduate School of Life Science, University of Hyogo, 3-2-1, Kouto, Kamigori-cho, Ako-gun, Hyogo 678-1297, Japan. ${ }^{9}$ Bioinformatics Laboratory, Niigata University Graduate School of Medical and Dental Sciences, Chuo-ku, Niigata 951-8510, Japan. ${ }^{10}$ Department of Gene Expression Regulation, Institute of Development, Aging and Cancer, Tohoku University, Sendai 980-8575, Japan. ${ }^{11}$ Department of Biological Sciences and Biotechnology, College of Life Sciences and Nanotechnology, Hannam University, Daejeon 34430, Korea. ${ }^{12}$ Department of Pharmaceutical Sciences, Faculty of Pharmacy, Keio University, Minato-ku 105-8512 Tokyo, Japan. 13 Institute of Biomedicine, University of Turku, Turku FI-20014, Finland. ${ }^{14}$ These authors contributed equally: Shun Kageyama, Sigurdur Runar Gudmundsson.

凶email: eeva-liisa.eskelinen@utu.fi; mkomatsu@juntendo.ac.jp
} 
$\mathrm{M}$ acroautophagy (thereafter referred to as autophagy) is defined as intracellular protein degradation pathway in lysosome $e^{1,2}$. The initial step of autophagy is the development of a single membrane sac, called isolation membrane/ phagophore, close to endoplasmic reticulum (ER). The isolation membrane/phagophore sequesters a part of cytoplasm, forming a double membrane structure, autophagosome ${ }^{3}$. The autophagosome fuses with a lysosome to degrade the sequestered materials into building blocks such as amino acids. There are two modes of autophagy: bulk and selective autophagy ${ }^{4}$. While the former is responsible for metabolic adaptation, the latter one contributes to cellular homeostasis ${ }^{5}$. Actually, mice defective in autophagy are sensitive to metabolic stress and also suffer from cell degeneration due to the accumulation of misfolded proteins and damaged organelles $^{6}$. Both modes are driven by core Autophagy-related (ATG) proteins, but a definite difference among them is that either the autophagosome randomly sequesters cytoplasmic components or it forms along specific cargos ${ }^{4}$. Specific autophagic cargos such as liquid droplets, damaged or excess organelles and aggregated proteins appear in the cytoplasm and are subsequently tagged with ubiquitin, leading to assembly of adaptor proteins such as p62, NBR1, NDP52, OPTN, and TAX1BP1 that bind to the ubiquitin chain ${ }^{7}$. Alternatively, transmembrane type of adaptor proteins, such as NIX, FAM134, and TEX264, directly localize on cargos ${ }^{7}$. In the case of the transmembrane adaptors, molecular markers like ubiquitin are not needed. Core autophagy-related proteins such as FAK family kinase-interacting protein of $200 \mathrm{kDa}$ (FIP200) also recognize the labelled targets through the interaction with adaptor proteins ${ }^{8-10}$, beginning the process of autophagosome formation around the targets. Ubiquitin-binding and transmembrane type adaptor proteins, both have LC3-interacting region(s)/GABARAP-interaction motif(s), called LIR/GIM to interact with autophagosomelocalizing ATG8 family proteins (including LC3A, LC3B, LC3C, gamma-aminobutyric acid receptor-associated protein (GABARAP), gamma-aminobutyric acid receptor-associated protein-like (GABARAPL)1, and GABARAPL2) around the cargos $^{11}$. To date, gene targeting for Atg genes revealed various physiological functions of autophagy ${ }^{12}$, but the membrane dynamics and physiological roles focusing on selective autophagy specifically are not well known. Herein, we investigated the properties of endogenous $\mathrm{p} 62 / \mathrm{SQSTM} 1$ (thereafter referred to as p62)-structures, which are a representative of a selective substrate for autophagy. We clarified the membrane dynamics of isolation membranes/phagophores targeting the p62-structures as well as the in vivo significance of autophagic turnover of the p62structures.

\section{Results}

p62-positive structures in cells are gels. Recent studies revealed that liquid droplets, such as Apel complex, stress granule, Pbody, and p62-body, which are formed by liquid-liquid phase separation, are selectively degraded by autophagy ${ }^{13-19}$. In the case of selective autophagy for Apel complex, P-body, and stress granule ${ }^{13,16,20}$, liquidity or interacting protein(s) have been shown to be crucial factors for the strucrutes to be surrounded by an autophagosome. Despite p62 is a typical selective substrate for autophagy and forms round structures with a range from 1 to 5 $\mu \mathrm{m}$ within cytoplasm $^{21}$, the dynamics of endogenous $\mathrm{p} 62$ structures still remains unknown. To investigate the properties and dynamics of endogenous p62-structures and their isolation by autophagosomes in cells, we utilized a hepatocellular carcinoma cell line, Huh-1, in which endogenous p62 expresses at high level ${ }^{22}$. Huh-1 cells possessed a large number of the p62-positive structures in the cytoplasm (Fig. 1a). Such structures were round with a diameter from 0.5 to $3 \mu \mathrm{m}$ and completely colocalized with the Neighbor of BRCA1 gene 1 protein (NBR1), a binding partner of p62 $2^{23}$ (Fig. 1a). p62 is phosphorylated at Ser403 (Ser405 in mice ${ }^{24}$ and Ser349 (Ser351 in mice) ${ }^{25}$ to enhance the binding to ubiquitinated proteins and to recruit kelch-like ECH-associated protein 1 (Keap1) on the p62-structures, respectively. The p62positive structures in Huh-1 cells contained both phosphorylated forms and were also positive for ubiquitin and Keap1 (Fig. 1a). Time lapse imaging of Huh-1 cells expressing GFP-p62 indicated that the p62-bodies have a spherical shape, move through the cytoplasm and occasionally fuse with each other (Supplementary Movie S1), matching with the criteria of liquid droplets and/or gels $^{26}$. We next purified the p62-structures from GFP-p62 expressing Huh-1 cells and then treated them with 1,6-hexanediol, an aliphatic alcohol that has been used to differentiate between liquid droplets and aggregates ${ }^{27}$. The structures dispersed within $5 \mathrm{~min}$ after the treatment (Fig. $1 \mathrm{~b}$ and Supplementary Movie S2). Fluorescence recovery after photobleaching analysis (FRAP) showed that average fluorescence recovery time after photobleaching of GFP-p62-labelled p62-structures is $9.2 \pm$ $1.2 \mathrm{~min}$ (Fig. 1c and Supplementary Movie S3). Considering that the recovery times are much slower compared with that of typical liquid droplets (scales of seconds to minutes) ${ }^{17,18,26}$, we concluded that the p62-structures in Huh-1 cells are gels.

p62-gels are degraded by autophagy. Core ATG proteins essential for autophagosome formation, such as FIP200, ULK1, WIPI2, and ATG16L1, were recruited to the p62-positive gels (Fig. 1d), implying autophagosome formation is constantly primed around the gels. Actually, treatment with Bafilomycin $A_{1}$ $\left(\mathrm{BafA}_{1}\right)$, which is an inhibitor of lysosomal acidification, significantly increased the levels of p62, S349- and S403phosphorylated p62 and NBR1 (Fig. 1e). These fluctuations were completely consistent with those of LC3-II and GABARAPII (Fig. 1e). Likewise, p62-gels were also observed in mouse embryonic fibroblasts (MEFs) isolated from p62-GFP knockin mice $\left(p 62-G F P^{K I /+}\right)$, in which C-terminal GFP-tagged p62 is driven by endogenous $\mathrm{p} 62$ regulatory elements ${ }^{28}$ (Supplementary Fig. S1). As p62 is a stress-inducible protein regulated by the Nuclear factor erythroid 2-related factor $2(\mathrm{Nrf2})^{29}$, a number of p62-GFP positive structures were formed in $p 62-G F P^{K I} /+\mathrm{MEFs}$ upon exposure to sodium arsenite (As[III]) (Supplementary Fig. S1a). They were round, contained the S351- and S405phosphorylated $\mathrm{p} 62$ forms and extensively colocalized with Keap1 and ubiquitin (Supplementary Fig. S1a). We confirmed similar properties of $\mathrm{p} 62$-structures in both mouse primary culture hepatocytes and HeLa cells after treatment with As[III] (Supplementary Fig. S1b, c), implying general properties of p62-gels. Consistent with previous report ${ }^{28}$, we verified the increased levels of p62-GFP and p62 in p62-GFPKI/+ MEFs under the As[III]treated conditions, which decreased after the removal of As[III] in a time-dependent manner (Supplementary Fig. S1d). Such decrease was abrogated by loss of Atg7, an essential gene for autophagy (Supplementary Fig. S1d), suggesting autophagic degradation of the p62-gels. Indeed, $19.5 \%$ of p62-gels in p62GFPKI/+ MEFs colocalized with WIPI2, an isolation membrane/ phagophore marker, $6 \mathrm{hr}$ after removal of As[III] (Supplementary Fig. S1e). Time lapse imaging of $p 62-G F P^{K I /+}$ MEFs indicated that after removal of As[III], p62-positive structures in wild-type background gradually decreased and most of them disappeared by $12 \mathrm{~h}$ (Supplementary Movie S4). By contrast, in Atg7-knockout background, such structures remained and rather became larger due to fusion of several structures with each other (Supplementary Movie S5). Taken together, these results indicate the autophagic degradation of p62-gels. 
a
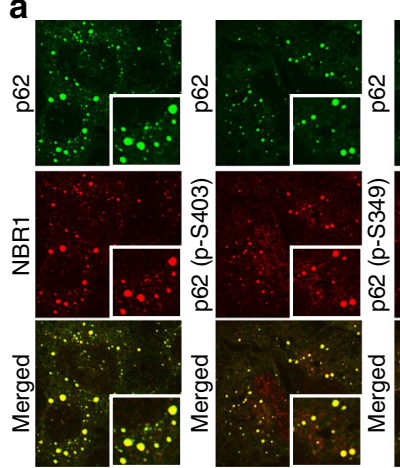

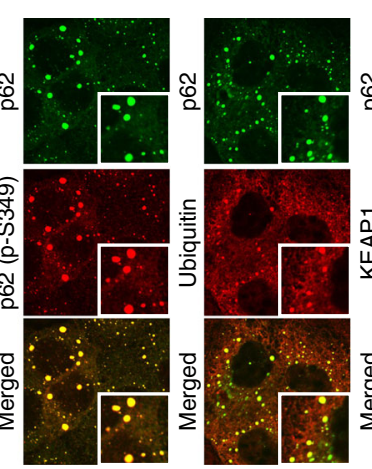

b

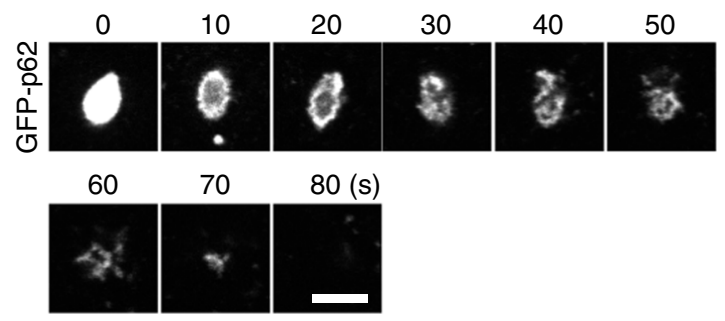

C
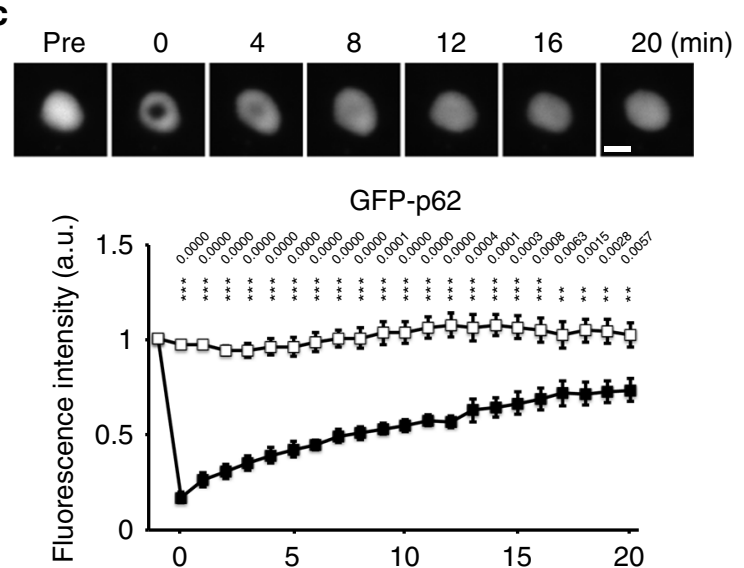

d
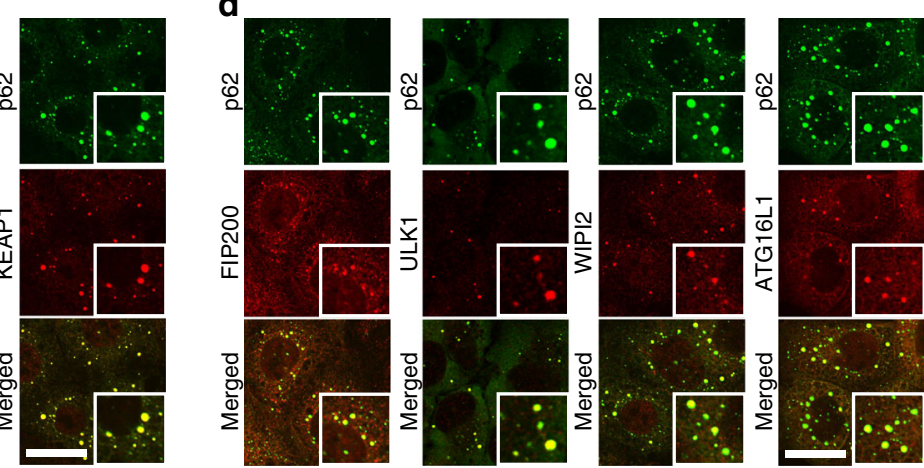

e

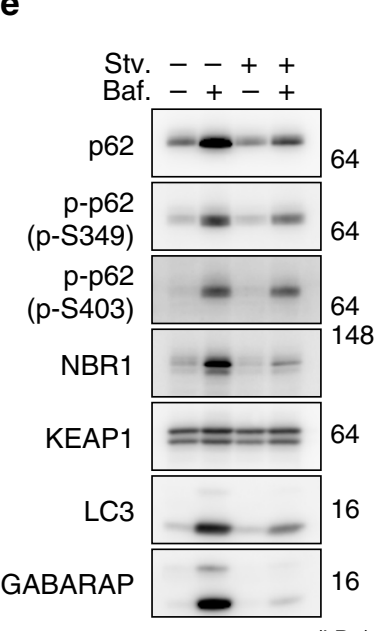

(kDa)

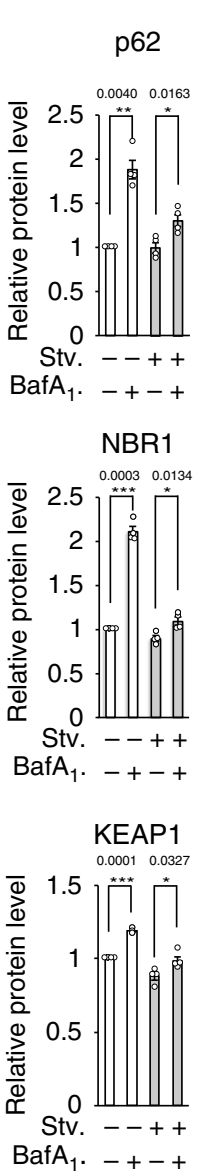

Fig. 1 p62-gels are degraded by autophagy. a Immunofluorescence microscopy. Huh-1 cells were immunostained with indicated antibodies. Bars: $10 \mu$ m. b Time lapse microscopic analysis. Huh-1 cells were transfected with GFP-p62. $24 \mathrm{~h}$ after the transfection, the p62-bodies labelled GFP-p62 were isolated from the cells and treated with 1,6-hexanediol. Bar: $5 \mu \mathrm{m}$. c Time lapse microscopic analysis. Huh-1 cells were infected by GFP-p62. $24 \mathrm{~h}$ after the infection, the p62-bodies labelled by GFP-p62 were photobleached, and then time of fluorescent recovery was measured. Data are means \pm s.e of nonphotobleached $(n=7)$ and photobleached $(n=10)$ dots. ${ }^{\star \star} p<0.01$, and ${ }^{\star \star \star} p<0.001$ as determined by two-sided Welch's $t$-test. Bar: $2 \mu \mathrm{m}$. Source data are provided as a Source Data file. d Immunofluorescence microscopy. Huh-1 cells were immunostained with indicated antibodies. Bar: $10 \mu \mathrm{m}$. e Immunoblot analysis. Huh-1 cells were cultured in regular medium in the presence or absence of $100 \mathrm{nM}$ Bafilomycin $\mathrm{A}_{1}$ (Baf.) for $24 \mathrm{~h}$ or cultured in amino acid-deprived medium (Stv.) for $4 \mathrm{~h}$ in presence or absence of Baf. Cell lysates were prepared and subjected to immunoblot analysis with the indicated antibodies. Data shown are representative of four separate experiments. Bar graphs indicate the quantitative densitometric analysis of the indicated proteins relative to whole proteins estimated by Ponceau-S staining $(n=4)$. Data are means \pm s.e. ${ }^{\star} p<0.05,{ }^{\star \star} p<0.01$, and ${ }^{\star \star \star} p<0.001$ as determined by two-sided Welch's $t$-test. Source data are provided as a Source Data file.

Autophagosome formation on p62-gels. Correlative lightelectron microscopy (CLEM) was performed to visualize the autophagy events during clearance of p62-GFP gels in p62-GFPKI/+ MEFs $3 \mathrm{~h}$ after removal of As[III]. Notably, in electron microscopy samples, even without correlation with GFP fluorescence, p62-GFP gels could be identified by their unique morphology (Fig. 2a-c), which has also been reported before ${ }^{30}$. In agreement with immunofluorescence analysis with WIPI2 antibody (Supplementary Fig. S1e), the p62-GFP gels were frequently observed inside isolation membranes/phagophores and autophagosomes, which in many cases had multiple double membranes on top of each other around them (Fig. 2a-f). Three-dimensional reconstructions were 

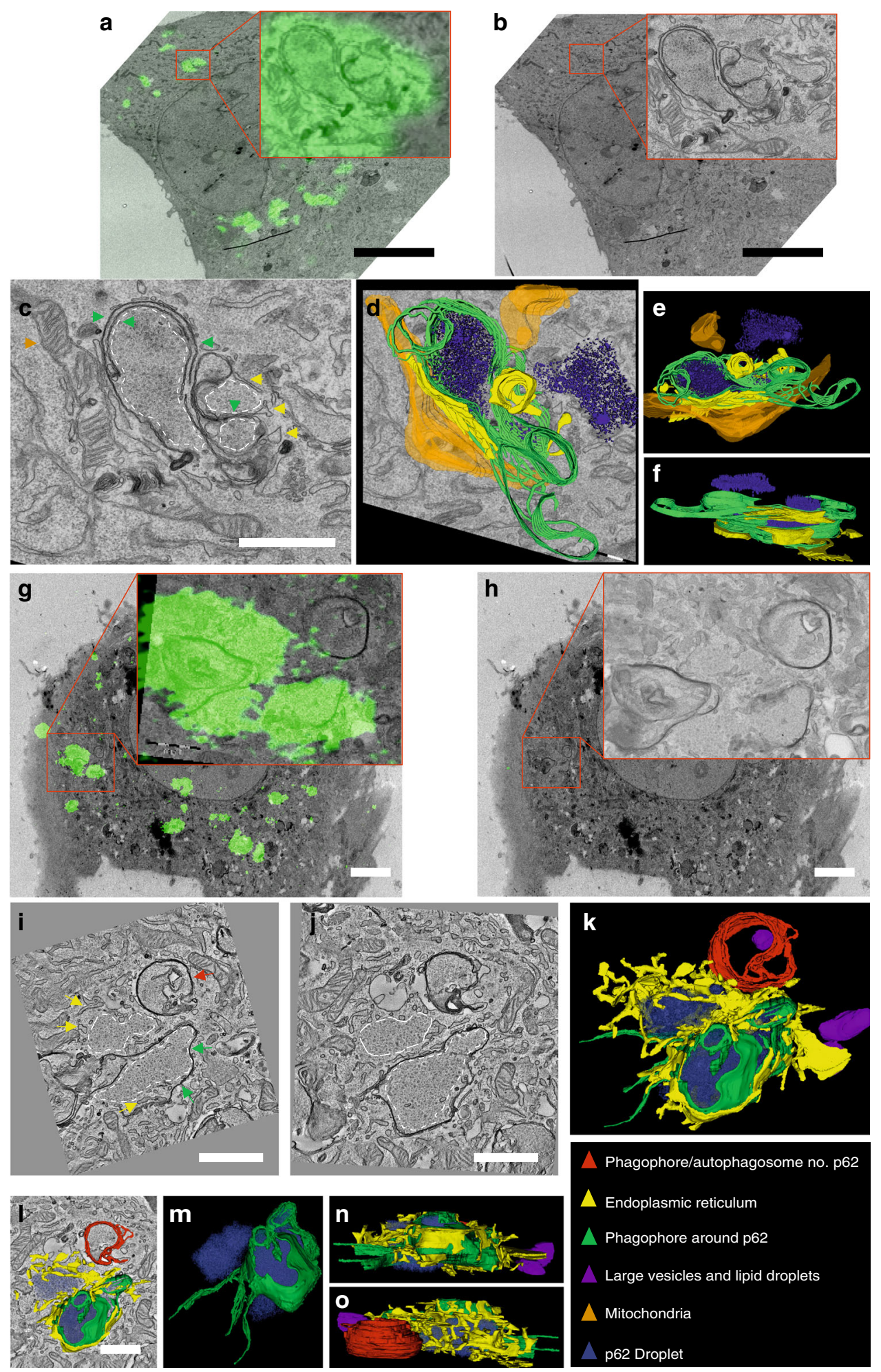

Fig. 2 Ultrastructure and three-dimensional models of autophagic membranes around p62-gels. a-f 80-nm TEM serial sectioning. p62-GFPKI/ + MEFs were treated by sodium arsenite (As[III]) for $10 \mathrm{~h}$ and then cultured in regular medium for $3 \mathrm{~h}$. $\mathbf{a}$, $\mathbf{b}$ Correlation of GFP fluorescence and TEM. p62-gels, identified by their unique morphology and by correlation with the GFP fluorescence, are indicated by dotted lines in panels $\mathbf{c}, \mathbf{i}$ and $\mathbf{j}$. Bars: $10 \mu \mathrm{m}$. $\mathbf{c}$ The p62gels are surrounded by single or multiple isolation membranes/phagophores or autophagosome limiting membranes (green arrows). Endoplasmic reticulum (yellow arrows) is observed next to the p62-gels and isolation membranes/phagophores or autophagosomes. Bar: $1 \mu \mathrm{m}$. Panels $\mathbf{d - f}$ show a threedimensional model segmented using aligned $80-\mathrm{nm}$ TEM serial sections. The color code is shown in the bottom right panel. $\mathbf{g}$, $\mathbf{h}$ Correlation of GFP fluorescence and a 200-nm TEM section used for tomography. Bars: $5 \mu \mathrm{m}$. i, j Single views from the electron tomogram, showing p62-gels (dotted lines), an isolation membrane/phagophore containing p62-gel (green arrows), and an isolation membrane/phagophore or autophagosome that does not contain p62-gel (red arrow). Bars: $0.5 \mu \mathrm{m}$. Panels k-o show a three-dimensional model segmented using four aligned 200-nm electron tomograms. The color code is shown in the bottom right panel. 
prepared using serial sections and electron tomography (Fig. 2c-o), and they demonstrated the unusually complex morphology of the isolation membranes/phagophores and autophagosome limiting membranes. Endoplasmic reticulum (ER) was frequently observed to surround the p62-GFP gels (Fig. 2c-f, i-o). Isolation membranes/phagophores and autophagosomes often segregated a p62GFP gel as a whole (Fig. 2c-e). In addition, autophagosomes and isolation membranes/phagophores were frequently observed next to p62-GFP gels, but not segregating the gel (Fig. 2i-l). Approximately $50 \%$ (49 out of 99 ) of the phagophores/isolation membranes and autophagosomes locating next to p62-gels were enveloping p62-gel, while the rest were enveloping other cytoplasmic components. Similar to the autophagy-intact MEFs, also in Atg7 $7^{-1-}$; p62-GFPKI/+ MEFs, ER was typically surrounding the p62-GFP gels (Supplementary Fig. S2b, c). In agreement with the immunoblot data (Supplementary Fig. S1d), only very few isolation membranes/phagophores and autophagosomes were observed in Atg7 ${ }^{-1-}$; p62-GFPKI/+ MEFs, and none of them segregated p62GFP gels (Supplementary Fig. S2e). On the basis of the morphological analysis, we concluded that there are two distinct ways of autophagosome formation on the p62-gels, either the p62-gel is engulfed by the forming autophagosome, or the autophagosome forms next to the p62-gel but does not engulf it. These two types of segregation probably correspond to selective and bulk autophagy.

Engulfment of p62-gels by autophagosome is dependent on LC3/GABARAP interaction. Which factor(s) decide(s) between the two distinct ways of autophagosome formation on the p62gels? We hypothesized that the interaction of p62 with LC3 and GABARAP is critical; if p62 interacts with LC3 and GABARAP, p62-gel is engulfed by the autophagosome, if not, autophagosomes form next to the p62-gel but do not engulf it. To test our hypothesis, we initially conducted an in vitro analysis using purified recombinant proteins and giant unilamellar vesicles (GUVs). Consistent with previous reports ${ }^{17}$, mixing of mCherryp62 with linear tetraubiquitin $(4 \mathrm{xUb})$ resulted in the formation of condensates, which was not affected by LIR mutation (W338A, L341A) in p62 (Fig. 3a). We then prepared Atg8-GUVs that contain Atg8-phosphatidylethanolamine conjugates by incubating GUVs with the Atg8 conjugation system and ATP, which were previously utilized to analyze the interaction with Ape1 condensates $^{13}$. The Atg8-GUVs were used for interaction analysis with p62-4xUb condensates since the ability to bind LIR motifs is evolutionarily conserved among Atg8 family proteins ${ }^{31}$. When p62-4xUb condensates were incubated with Atg8-GUVs, both dispersed p62 and 4xUb and their condensates were bound to Atg8-GUVs. Bound condensates were confirmed not to be membranous structures using GUVs with fluorescently labeled lipids (Fig. 3b). On the other hand, both of them were only scarcely bound to Atg8-GUVs when W338A, L341A mutation was introduced to p62 (Fig. 3b, c). These data indicate that p62$4 \mathrm{xUb}$ condensates interact with Atg8-GUVs depending on the LIR-Atg8 interaction, which is consistent with previous report ${ }^{32}$, proposing that p62-gels could be tethered to isolation membranes via LIR-LC3/GABARAP interaction.

In the next experiments, we sought to clarify this issue in vivo. p62 LIR mutants were not used since a recent report showed that the LIR of p62 overlaps with an interacting region of FIP200, which is an upstream ATG protein ${ }^{8}$. Instead, we utilized a HyDLIR-Venus construct, consisting of $\mathrm{HyD}$, a short hydrophobic domain of Aplysia phosphodiesterase 4 short-form; LIR of TP53INP2; and a fluorescent protein, Venus ${ }^{33}$. This protein efficiently binds to LC3-II and GABARAP-II localizing on the inner membrane of autophagosomes and does not affect autophagosome formation ${ }^{33}$ (Fig. 4a). If the interaction of p62 with LC3-II and/or GABARAP-II directs the isolation membranes/phagophores to the p62-gels, the overexpression of $\mathrm{HyD}$ LIR-Venus should inhibit autophagosome sequestration of p62gels by the competitive inhibition (Fig. 4a). We generated knockin mice that express HyD-LIR-Venus under CAG promoter in a Cre-recombinase-dependent manner (HyD-LIR flox/flox) (Supplementary Fig. S3). HyD-LIR flox/flox mice were crossbred with AlbCre transgenic mice ${ }^{34}$ that express the Cre recombinase under the control of the Albumin promoter to generate hepatocyte-specific HyD-LIR-Venus expressing mice (HyD-LIRflox/flox; Alb-Cre). As expected, marked expression of HyD-LIR-Venus protein was recognized in primary hepatocytes isolated from $H y D-L I R^{\text {flox/flox; }}$ Alb-Cre but not control HyD-LIR flox/flox mice (Fig. 4b). The expression of HyD-LIR-Venus hardly affected the conversion of LC3-I to LC3-II, rather it increased the levels of LC3-II (Fig. 4b). Treatment with $\mathrm{BafA}_{1}$ increased the amount of LC3-II in both control and HyD-LIR expressing hepatocytes (Fig. 4b). Due to high expression, we did not observe any fluctuation of HyD-LIRVenus by treatment with $\mathrm{BafA}_{1}$. Immunofluorescence analysis with LC3 antibody showed colocalization of HyD-LIR-Venus with LC3-positive structures (Fig. 4c). In agreement with the biochemical analysis, the number of LC3-positive puncta in hepatocytes expressing HyD-LIR-Venus was much higher than that in control hepatocytes (Fig. 4c). Whereas the LC3-positive dots increased in number upon nutrient deprivation in control hepatocytes, such increase in the HyD-LIR-Venus expressing hepatocytes was milder (Fig. 4c), due to large number even in nutrient-rich conditions.

We observed a prominent accumulation of p62 in HyD-LIR expressing hepatocytes regardless of nutrient conditions, which was not further increased by treatment with BafA 1 (Fig. 4b). The treatment with $\mathrm{BafA}_{1}$ slightly but significantly upregulated the amount of S351-phosphorylated p62 and its client proteins, NBR1 and Keap1 (Fig. 4b). Immunofluorescence analysis showed a prominent accumulation of p62-positive structures in hepatocytes overexpressing HyD-LIR-Venus (Fig. 4c). Although we detected p62-positive structures in control hepatocytes, their size and number were smaller than those in HyD-LIR-Venus overexpressing hepatocytes (Fig. 4c). We developed adenovirus vector expressing HyD-LIR-Venus, a tool that enabled easy expression of HyD-LIR-Venus regardless of cell types at high level. Similar to HyD-LIR-Venus expressing hepatocytes, p62positive structures were formed by simple infection of HeLa cells with the HyD-LIR-Venus adenovirus, and they showed spherical shape (Supplementary Fig. S4). To investigate if these p62structures exhibit gel-like or aggregate-properties, we carried out time lapse imaging. As shown in Supplementary Movie S6, the p62-structures moved through the cytoplasm and occasionally fused with each other. These results indicate that the p62-positive structures formed by the expression of HyD-LIR-Venus are liquid droplets and/or gels.

WIPI2, a core autophagy-related protein, extensively colocalized with the p62-gels in both control and HyD-LIR Venus expressing hepatocytes (Fig. 4d), implying autophagosome formation on the p62-gels. We observed signals for HyD-LIRVenus next to or overlapping with the p62-gels (Fig. 4c). Immunoelectron microscopy (IEM) of HyD-LIR Venus expressing hepatocytes revealed that p62-gels with round or elliptical shape contained numerous vesicles and electron-dense materials (Fig. 4e). Similar structures with diameters of approximately $1 \mu \mathrm{m}$ were often observed by conventional electron microscopy. (Fig. 4f). Autophagosome/phagophore profiles were occasionally observed in proximity of the p62-gels, but they very rarely enwrapped the gels (Fig. 4f). We have also provided the following evidence: LC3-positive puncta are colocalized with p62structures $^{35}$, p62-structures consisting of LC3/GABARAP- 
a

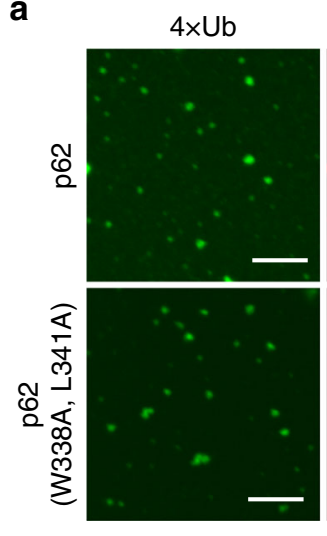

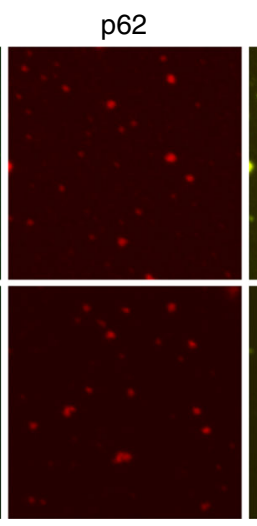

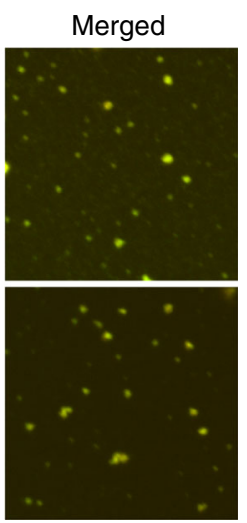

C

b
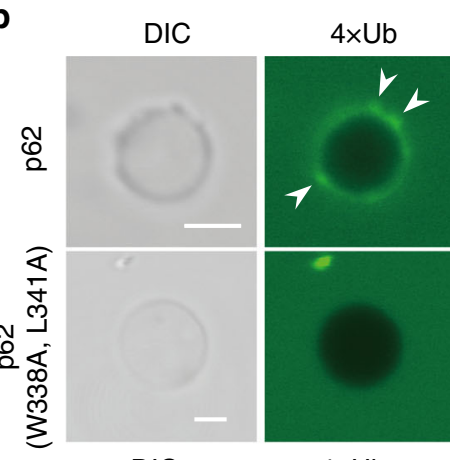

$4 \times \mathrm{Ub}$

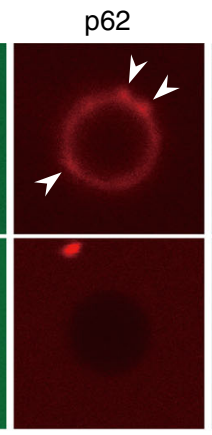

p62

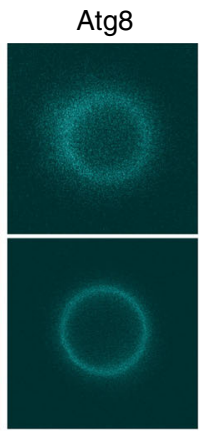

Membrane
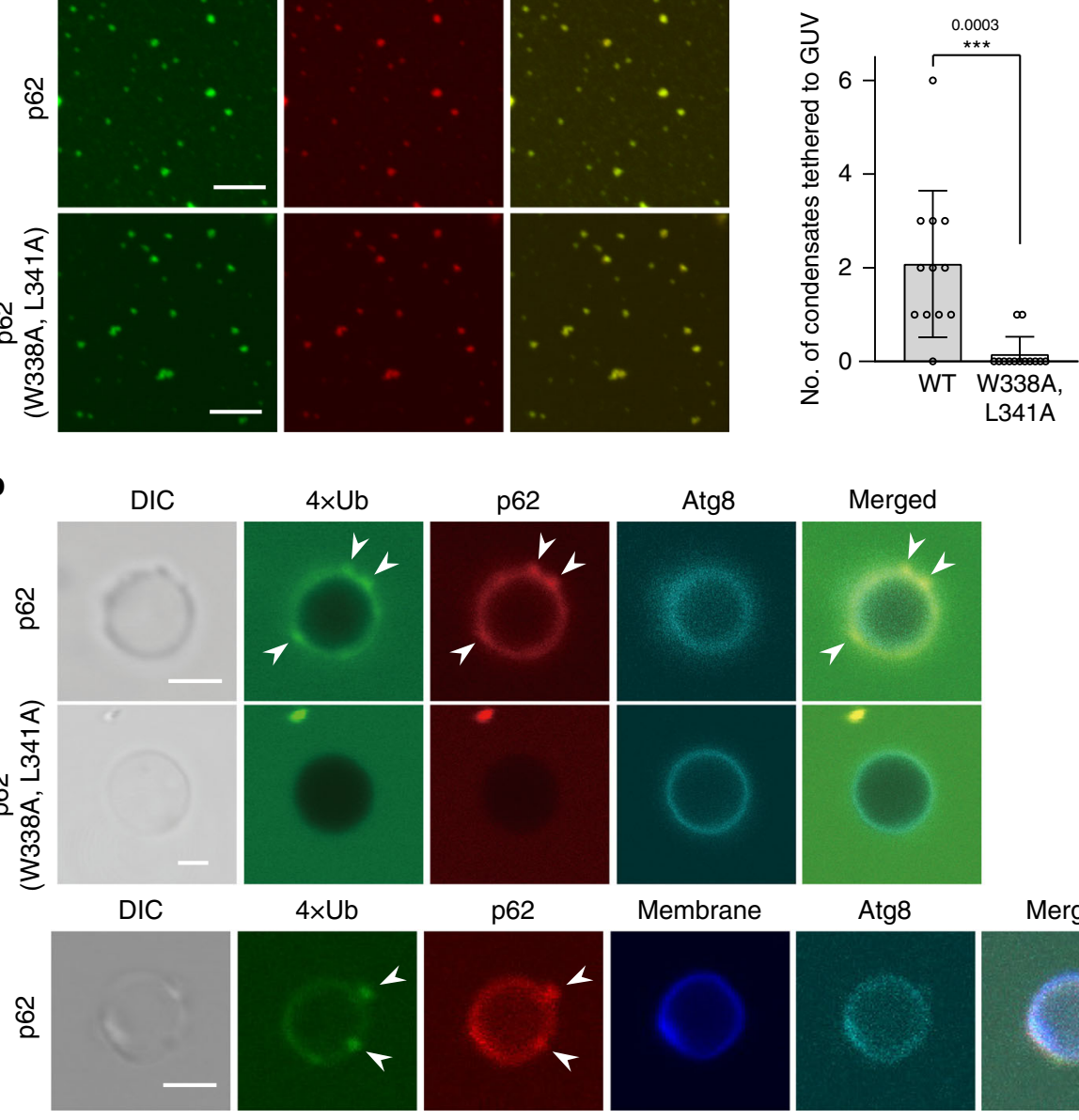

Fig. 3 p62-4xUb condensates interact with Atg8-GUVs depending on the LIR-Atg8 interaction. a In vitro formation of p62-4xUb condensates. $5 \mu \mathrm{M}$

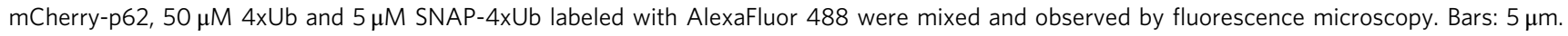
b Interaction analysis of p62-4xUb condensates with Atg8-GUVs. p62-4xUb condensates as prepared in a were incubated with mKalama1-Atg8-GUVs and observed by fluorescence microscopy. DIC differential interference contrast. Arrowheads indicate p62-4xUb condensates on the Atg8-GUV. Bars: $2 \mu \mathrm{m}$. c Quantification of the experiments in b. GUVs were randomly selected ( $n=12$ and 13 for WT and W338A L341A, respectively) and the number of attached condensates was counted. Data are means \pm s.d of wild-type and W338A L341A condensates attached with a GUV. ${ }^{\star \star \star} P<0.001$ as determined by two-sided Student's $t$-test. Source data are provided as a Source Data file.

binding defective p62 are not degraded by autophagy ${ }^{36}$, and phagophore profiles surround p62-body as shown by $\mathrm{IEM}^{37}$. On the basis of the published and presented evidence, we concluded that that autophagosome formation occurs on the p62-gels and that the interaction of p62 with LC3 and/or GABARAP directs the process towards the engulfment of whole p62-gels by autophagosomes.

Impairment of LC3-interaction-dependent selective autophagy. Despite the presence of several autophagosomes and autolysosomes in the primary hepatocytes isolated from $H y D-L I R f l o x / f l o x$; Alb-Cre mice, the cells appeared to contain increased amounts of mitochondria and endoplasmic reticulum (Supplementary Fig. S5a-f). This observation implies defective mitochondriaturnover (mitophagy) and ER-turnover by autophagy (ERphagy). Indeed, we verified prominent accumulation of mitochondrial and ER-proteins, such as Tom20, Cytochrome b, and TEX264 in the hepatocytes (Supplementary Fig. S5g). Long-lived protein degradation assay was then used to assess autophagy and revealed significantly lower degradation in the $H y D-L I R$ flox/flox; Alb-Cre hepatocytes compared with that in control hepatocytes (Supplementary Fig. S5h). Nutrient deprivation induced protein degradation in hepatocytes of both genotypes, but the induction in the HyD-LIR expressing hepatocytes was significantly lower (Supplementary Fig. S5h). Protein degradation was suppressed to similar levels by the addition of lysosomal inhibitors, E64d, pepstatin A, and ammonium chloride (E64d/PepA + AC) in both genotypes (Supplementary Fig. S5h). The induced degradation in both genotypes was also inhibited by treatment with lactacystin, a proteasome inhibitor (Supplementary Fig. S5h). Considering that almost complete suppression of long-lived protein degradation has been observed in Atg7-deficient hepatocytes ${ }^{38}$, these results suggest that LC3/GABARAP-interaction-dependent selective autophagy is impaired in hepatocytes of $H y D-L I R^{\text {flox/flox; }}$, Alb-Cre mice, while bulk autophagy is intact.

Nrf2 activation on p62-gels. What is the biological significance of p62-gels? An in vitro analysis using purified recombinant proteins showed that Keap1 was recruited to p62-4xUb condensates (Fig. 5a). When the p62-4xUb-Keap1 condensates were incubated with Atg8-GUVs, they were bound to Atg8-GUVs (Fig. 5b). While GFP-p62 had an ability to form punctate structures in both wild-type and Keap1-deficient MEFs, structures positive for GFP-Keap1 were dispersed in p62-deficient MEFs 
a

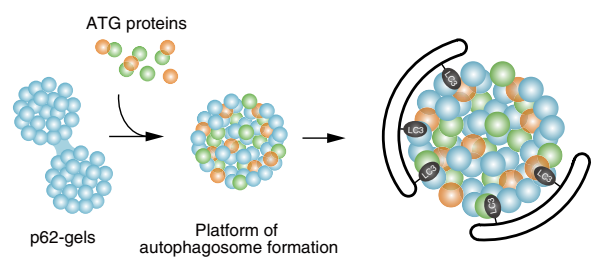

HyD-LIR-Venus

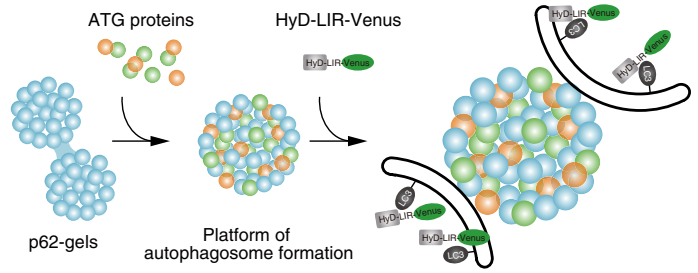

b
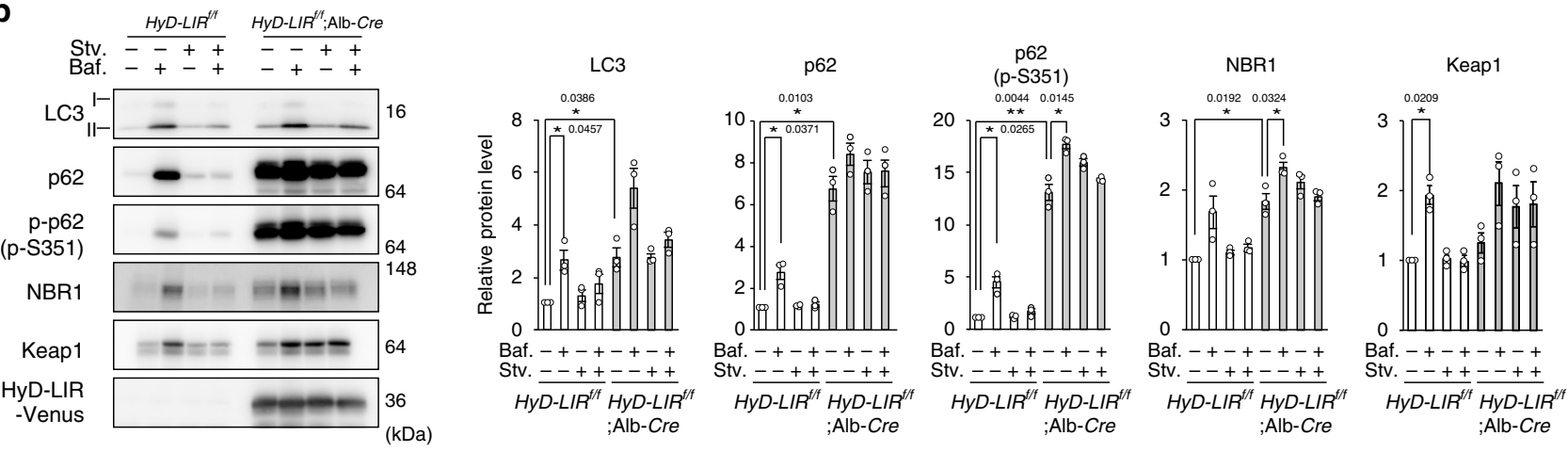

C
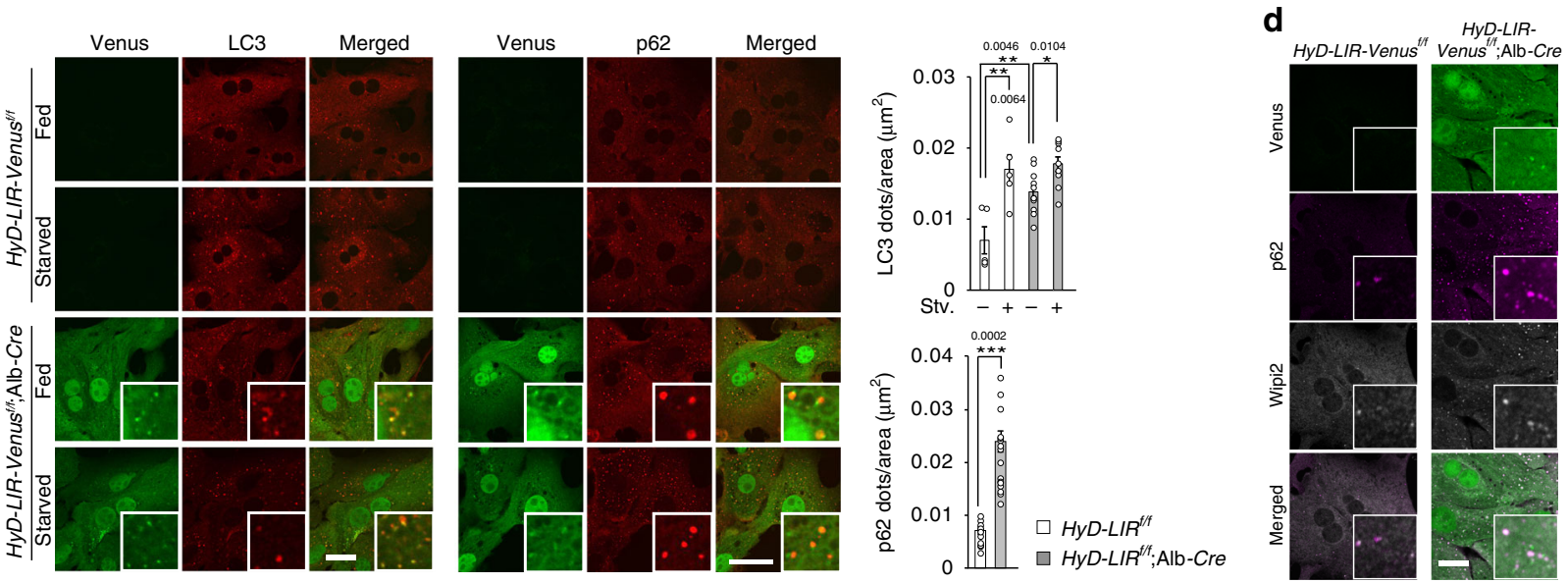

e
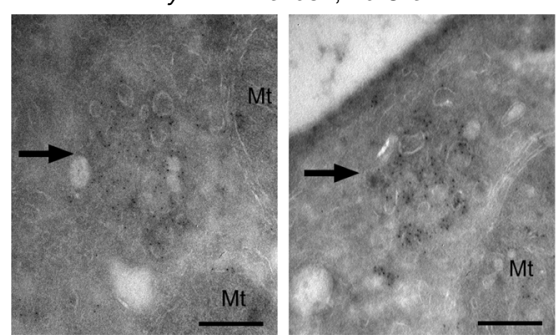

f

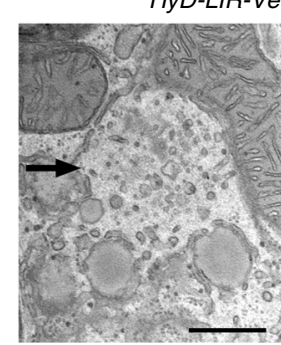

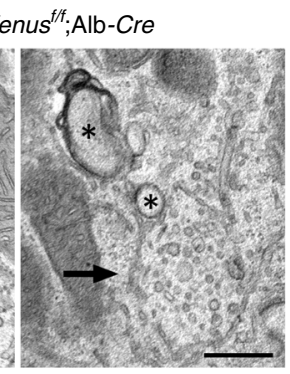

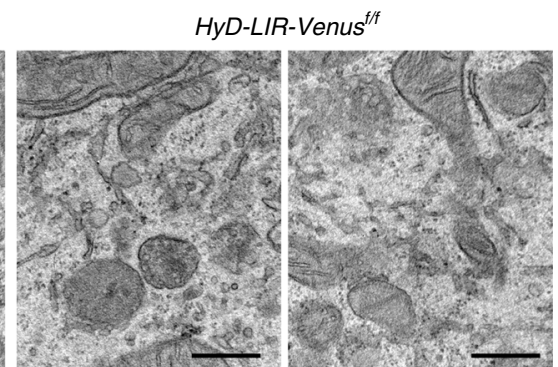

(Fig. 5c). These results suggest that Keap1 is translocated onto the p62-gels.

Keap1, an adaptor protein of cullin3-based ubiquitin ligase for Nrf2, interacts with S349-phosphorylated p62, and its binding mode is similar to that with Nrf2-ETGE motif ${ }^{25,39}$. We hypothesized that the p62-gel acts as a platform for Nrf2 activation through the sequestration of Keap1 onto the gel. To study this possibility, we used a chemical compound termed $\mathrm{KMNO03}^{40}$ (Fig. 6a), which is a derivative of K67. K67 is a Keap1binding chemical compound that inhibits the interaction of Keap1 with p62 $2^{40,41}$. The binding model of KMN003 with Keap1 was created based on the X-ray crystal structure of K67 in a complex with Keap1-DC domain consisting of the double glycine repeat or kelch repeat (DGR, aa 315-598) and the C-terminal region (CTR, aa 599-624) (Protein Data Bank ID code 4ZY3) ${ }^{41}$ using the CNS (crystallography and NMR system) energy minimization program ${ }^{42}$. The model showed that KMN003 is placed into the pocket at the bottom of the $\beta$-propeller structure essential for the interaction with p62 (Fig. 6b). The naphthalene ring, benzene rings and ethoxybenzene moieties of KMN003 fit into the pocket in the same manner as in the Keap1-DC-K67 complex (Fig. 6b). In addition, carboxyl group of side chain on the C-2 position of naphthalene ring interacts with R415 through a salt bridge (Fig. 6b), implying the higher inhibitory effect of 
Fig. 4 The interaction of $\mathbf{p 6 2}$ with LC3 directs autophagosomes toward the p62-gel. a Model of the effect of HyD-LIR-Venus. b Immunoblot analysis. Primary hepatocytes isolated from indicated genotype mice were cultured in regular medium in the presence or absence of $100 \mathrm{nM}$ Bafilomycin $\mathrm{A}_{1}$ (Baf.) for $24 \mathrm{~h}$ or cultured in amino acid-deprived medium for $4 \mathrm{~h}$ (Stv.) in presence or absence of Baf. Cell lysates were prepared and subjected to immunoblot analysis with the indicated antibodies. Data shown are representative of three separate experiments. Bar graphs indicate the quantitative densitometric analysis of the indicated proteins relative to whole proteins estimated by Ponceau-S staining $(n=3)$. Data are means \pm s.e. ${ }^{\star} P<0.05$ as determined by twosided Welch's $t$-test. Source data are provided as a Source Data file. c, d Immunofluorescence microscopy. Primary hepatocytes described in $\mathbf{b}$ were cultured in regular medium (Fed) (c, d) or amino acid-deprived medium for $4 \mathrm{~h}$ (Starved) (c). The cells were immunostained with indicated antibodies. The number of LC3- and p62-positive dots in hepatocyte was determined in each genotype. Data are means \pm s.e. of LC3-positive dots per area ( $\left.\mu m^{2}\right)$ in fed $(n=5)$ and starved HyD-LIRflox/flox $(n=5)$, fed $(n=11)$ and starved HyD-LIRflox/flox; Alb-Cre $(n=9)$ and p62-positive dots per area $\left(\mu m^{2}\right)$ in fed $(n=12)$ and starved HyD-LIRflox/flox $(n=16) .{ }^{\star} p<0.05,{ }^{\star \star} p<0.01$, and ${ }^{\star \star \star} p<0.001$ as determined by two-sided Welch's $t$-test. Bars: $20 \mu \mathrm{m}$. Source data are provided as a Source Data file. e Immunoelectron microscopy. Ultrathin-cryosections from the primary hepatocytes of indicated genotype cultured in regular medium were stained with p62 antibody (detected by colloidal gold particles of $6 \mathrm{~nm}$ in diameter). Arrows: p62-gel, Mt: mitochondria, Bar: $200 \mathrm{~nm}$. f Electron microscopy. Representative electron micrographs of the primary hepatocytes of indicated genotype cultured in regular medium are shown. Arrows: p62-gel, Asterisks: autophagosome/phagophore profiles, Bars: $500 \mathrm{~nm}$.

a

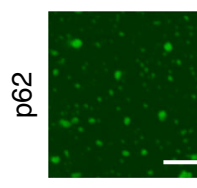

$4 \times \mathrm{Ub}$
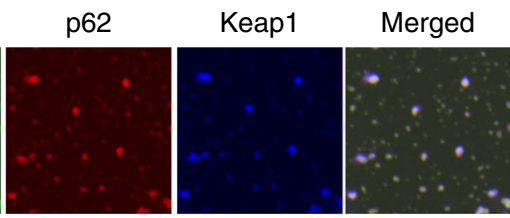

b

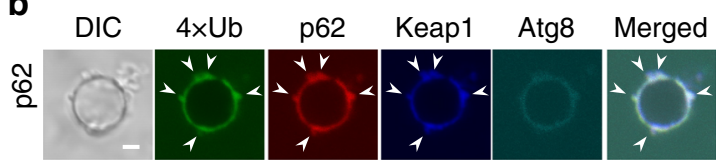

C

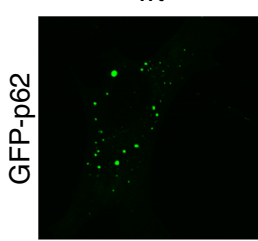

wt

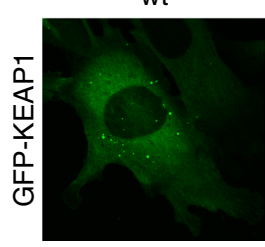

Keap1-KO

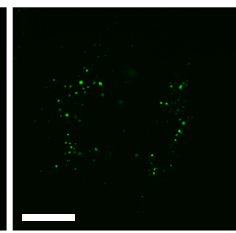

p62-KO

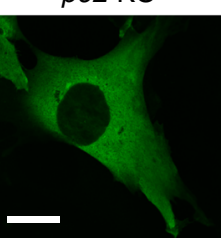

Fig. 5 Localization of Keap1 on the p62-gels. a In vitro formation of p62-4xUb-Keap1 condensates. $5 \mu$ M mCherry-p62, $50 \mu M \quad 4 x U b, 5 \mu M$ SNAP-4xUb labeled with AlexaFluor 488 and SNAP-Keap1 labelled with Alexa Fluor 647 were mixed and observed by fluorescence microscopy. Bars: $5 \mu \mathrm{m}$. b Interaction analysis of p62-4xUb-Keap1 condensates with Atg8-GUVs. p62-4xUb-Keap1 condensates as prepared in a were incubated with mKalama1Atg8-GUVs and observed by fluorescence microscopy. DIC differential interference contrast. Arrowheads indicate p62-4xUb-Keap1 condensates on the Atg8-GUV. Bars: $2 \mu \mathrm{m} \mu \mathrm{m}$. c Fluorescence microscopy. Wild-type, p62- or Keap1-knockout MEFs were transfected with GFP-p62 or GFP-Keap1. $24 \mathrm{~h}$ after the transfection, the cells were observed by confocal microscopy. Bars: $20 \mu \mathrm{m}$.

KMN003 to the p62-binding, compared to K67. Actually, in vitro pull-down assays revealed that KMN003 disturbed the interaction of Keap1-DC with S349-phosphorylated p62 at low concentration (Fig. 6c). Immunoprecipitation assay with Keap1 antibody showed that both p62 and S349-phosphorylated p62 were coimmunoprecipitated with Keap1, and both interactions decreased by the treatment of KMN003 in a dose-dependent manner (Fig. 6d). Treatment of Huh-1 cells with KMN003 increased the level of endogenous Keap1 (Fig. 6d), suggesting impairment of p62-mediated Keap1-turnover through autophagy ${ }^{43}$. Immunofluorescence analysis with p62 antibody revealed that the treatment of Huh-1 cells expressing GFP-Keap1 with KMN003 decreased the number of GFP-Keap1-positive p62-gels (Fig. 6e). Time lapse imaging of Huh-1 cells expressing GFP-Keap1 indicated that the GFP-Keap1-positive structures were round, moved through the cytoplasm and occasionally fused with each other similar to GFP-p62 (Supplementary Movie S7). FRAP analysis showed that after photobleaching, signal for GFP-Keap1labelled dots recovered and that the average recovery time was $2.67 \pm 0.44 \mathrm{~min}$ (Fig. $6 \mathrm{f}$ and Supplementary Movie S8), which is much faster than that of GFP-p62 (Fig. 1c and Supplementary Movie S3). We noticed that puncta positive for GFP-Keap1 often disappear upon the exposure to KMN003 (Supplementary Movie S9). While the GFP-Keap1 structures emerged in normal culture conditions (Supplementary Movie S7), we hardly observed formation of new GFP-Keap1-positive structures under the KMN003-treated conditions (Supplementary Movie S9). These results indicate that Keap1 is translocated to the p62-gels in a p62-binding dependent fashion and that the translocation is reversible.

We next investigated the significance of Keap1 on p62-gels. When wild-type p62 or T350A-p62 mutant, which does not bind to Keap $1^{39}$, was expressed in p62-deficient Huh-1 cells, both wildtype and mutant p62 formed similar punctate structure (Fig. 7a). Keap1 colocalized with wild-type but not mutant p62 (Fig. 7a). The expression of wild-type p62 caused nuclear accumulation of Nrf2, but the T350A mutant did not (Fig. 7b). As expected, the gene expression of Nrf2-targets, such as $N A D(P) H$ dehydrogenase, quinone 1 (Nqo1), and glutamate-cysteine ligase catalytic subunit $(\mathrm{Gclc})$, was markedly induced by overexpression of wild-type p62, but not the T350A mutant (Fig. 7c). We verified the increased level of Nqo1 and Gclc proteins in cells expressing wild-type p62, while such increase was not observed in cells harboring T350Ap62 (Fig. 7a). As expected, an oligomerization-defective K7A D69A mutant of p62 did not form p62-gels nor activate Nrf2 (Fig. 7a-c). Taken together, we concluded that the p62-gel acts as oxidative stress response through the sequestration of Keap1.

The combination of Nrf 2 activation with impaired autophagy causes liver injury. Similar to the primary hepatocytes (Fig. 4), p62, phosphorylated forms of p62, NBR1 and Keap1 accumulated 
a<smiles>CCOc1ccc(S(=O)(=O)Nc2cc(CCCC(=O)O)c(NS(=O)(=O)c3ccc(OCC)cc3)c3ccccc23)cc1</smiles>

C

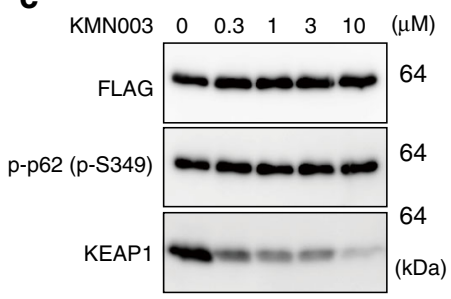

e
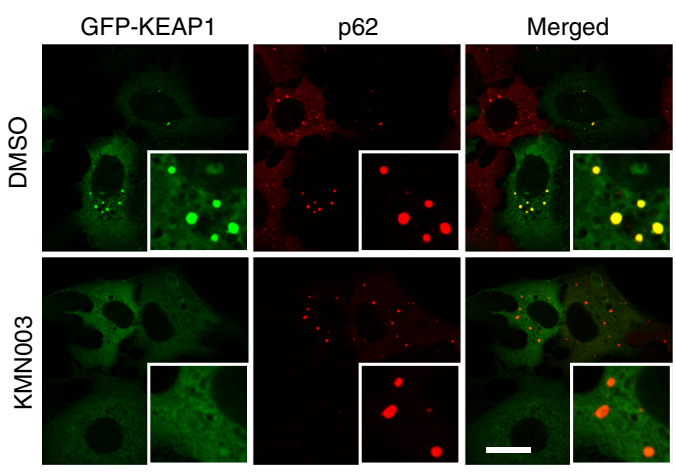

b

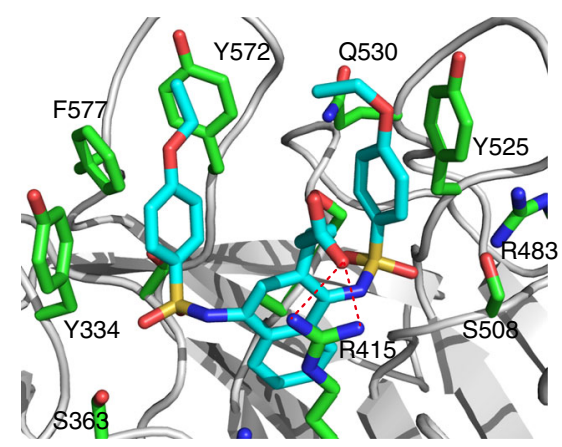

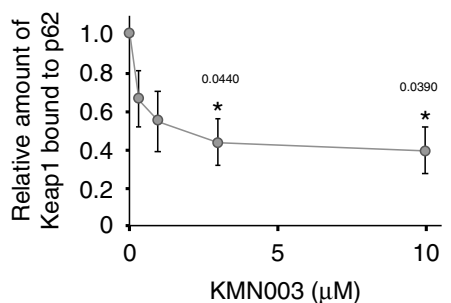

KMN003 $(\mu \mathrm{M})$

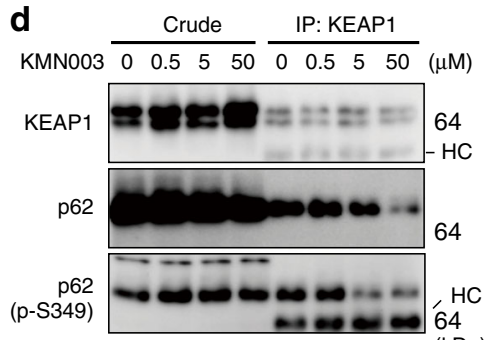

$(\mathrm{kDa})$

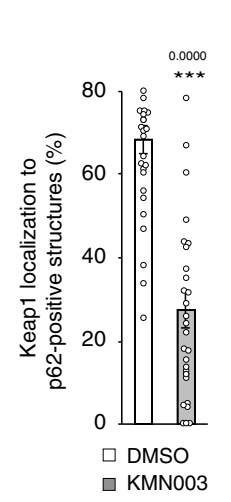

Fig. 6 Reversible translocation of Keap1 on the p62-gels. a Structural formulas of KMN003. (b) Modelling of Keap1 (white)-KMN003 (cyan). Overall structure of Keap1-DC, shown as a ribbon model. KMNO03 (cyan) and some of the potential interacting residues of Keap1-DC are shown in stick representation (green). Potential hydrogen-bonds are depicted as dotted red lines. $\mathbf{c}$ In vitro pull-down assay. Recombinant Keap1-DC was allowed to form a complex with Strep-FLAG-tag p62, and then KMN003 was mixed with the complex at indicated concentration. Keap1-DC binding to Strep-FLAG-tag p62 was estimated by immunoblot analysis with Keap1 antibody. Data are representative of three independent experiments. Right graph indicates the quantitative densitometric analysis of Keap1 bound to p62 $(n=3)$. Data are means \pm s.e. ${ }^{*} p<0.05$ as determined by two-sided Welch's $t$-test. Source data are provided as a Source Data file. d Immunoprecipitation assay. Huh-1 cells were cultured in regular medium in the presence or absence of KMN003 at indicated concentration for $12 \mathrm{~h}$. Cell lysates were immunoprecipitated with Keap1 antibody, and the immunoprecipitants were subjected to immunoblot analysis with the indicated antibodies. Data shown are representative of three separate experiments. HC indicates heavy chain. Source data are provided as a Source Data file. e Immunofluorescence microscopy. Huh-1 cells expressing GFP-Keap1 were cultured in regular medium in the presence or absence of 10 $\mu \mathrm{M} \mathrm{KMN003} \mathrm{for} 24 \mathrm{~h}$ and then immunostained with p62 antibody. Data are means \pm s.e. of the number of p62-positive dots with GFP-Keap1 in Huh-1 ( $n=$ 30). ${ }^{\star \star \star} P<0.001$ as determined by two-sided Welch's $t$-test. Bars: $20 \mu \mathrm{m}$. Source data are provided as a Source Data file. $\mathbf{f}$ Time lapse microscopic analysis. Huh-1 cells were transfected with GFP-Keap1. $24 \mathrm{~h}$ after the transfection, the structures labelled by GFP-Keap1 were photobleached, and then time of fluorescent recovery was measured. Data are means \pm s.e of nonphotobleached $(n=8)$ and photobleached $(n=10)$ dots. ${ }^{\star \star} p<0.01$, and ${ }^{\star \star \star} p<0.001$ as determined by two-sided Welch's $t$-test. Bar: $2 \mu \mathrm{m}$. Source data are provided as a Source Data file.

in livers of HyD-LIR flox/flox; Alb-Cre but not in livers of control mice (Fig. 8a). The levels were lower than those in livers of Atg 7 flox/flox; Alb-Cre mice (Fig. 8a). As shown in Fig. 3e, both p62 and Keap1 in hepatocytes expressing HyD-LIR-Venus were degraded in lysosomes although less efficiency than in control hepatocytes, suggesting that some of p62-gels are still surrounded by autophagosomes, likely by chance. Meanwhile, autophagosome formation is severely impaired in hepatocytes lacking Atg7, and the remnant Atg7-independent autophagosomes ${ }^{44,45}$ cannot engulf the p62-gels (Supplementary Fig. S2). Thus, it is reasonable that levels of p62 and Keap1 in hepatocytes expressing HyD-LIRVenus are lower than those in Atg7-deficient hepatocytes. We observed numerous punctate signals indicating p62-gels, which contained ubiquitinated proteins and S351-phosphorylated form of p62 in livers of both HyD-LIRflox/flox; Alb-Cre and Atgfflox/flox; Alb-Cre mice, but not in control livers (Fig. 8b). The p62-gels in HyD-LIR-Venus expressing hepatocytes tended to be smaller than those in Atg7-deficient hepatocytes, which occasionally contain large pleomorphic p62-aggregates (Fig. 8b). We speculated that Nrf2 is persistently activated in livers of $H y D-L I R^{\text {flox/flox; }}$ Alb- 
a

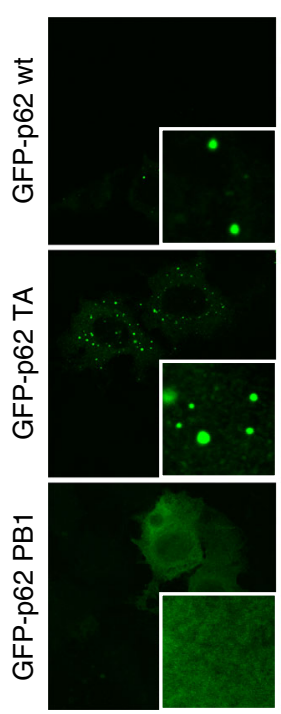

KEAP1
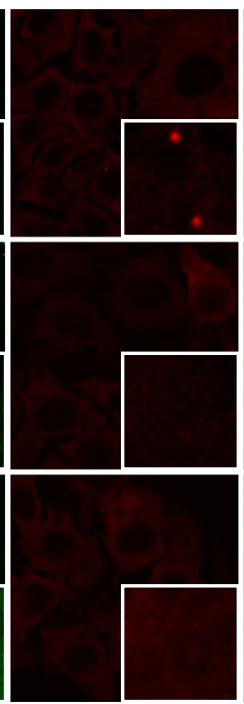

Merged

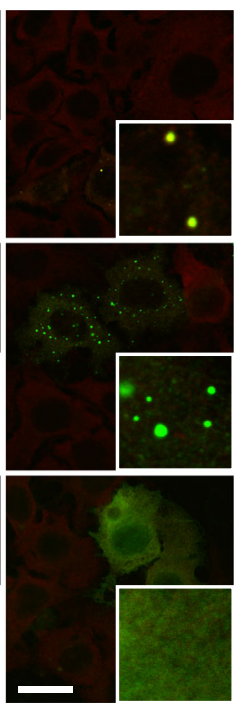

b
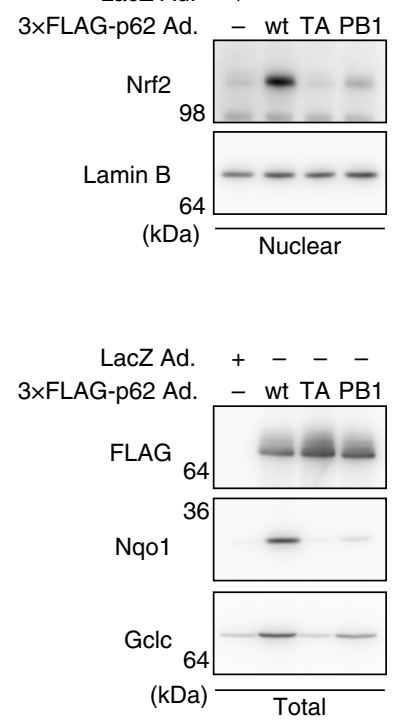

C

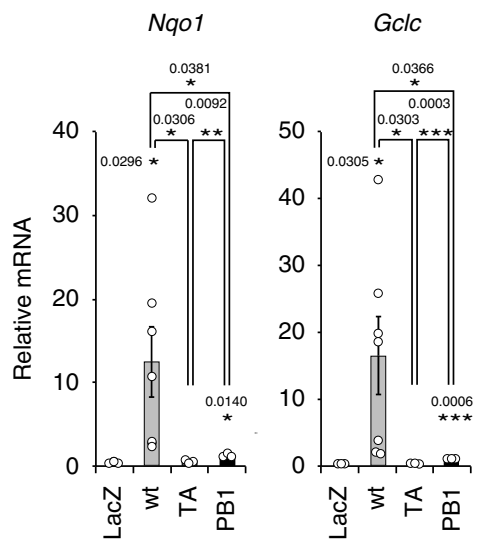

Fig. 7 Significance of Keap1 on p62-gels. a p62-deficient Huh-1 cells were transfected with wild-type p62, T350A or PB1 mutant. $24 \mathrm{~h}$ after the transfection, the cells were immunostained with Keap1 antibodies. Bar: $20 \mu \mathrm{m}$. b Wild-type p62, T350A or PB1 mutant was expressed in primary mouse hepatocytes using adenovirus system. $48 \mathrm{~h}$ after the infection, the cells were fractionated into cytosolic and nuclear fractions, which were subjected to immunoblot analysis with the indicated antibodies. Data shown are representative of three separate experiments. Source data are provided as a Source Data file. (c) Gene expression of Nrf2-targets. Total RNAs were prepared from primary mouse hepatocytes expressing LacZ $(n=7)$, wild-type p62 $(n=7)$, T350A $(n=7)$, or PB1 mutant $(n=3)$. Values were normalized against the amount of mRNA in LacZ-expressing hepatocytes. RT-qPCR analyses were performed as technical replicate on each biological sample. Data are means \pm s.e. ${ }^{\star} p<0.05,{ }^{\star \star} p<0.01$, and ${ }^{\star \star *} p<0.001$ as determined by two-sided Welch's t-test. Source data are provided as a Source Data file.

Cre mice due to the accumulation of the p62-gels. To test this, we carried out quantitative proteomics with livers of $H y D-L I R^{\text {flox/flox }}$ and $H y D-L I R^{f l o x} / f l o x$; Alb-Cre mice and found significant upregulation $(<1.5$-fold $)$ of 163 proteins including p62 in livers of $H y D$ LIR flox/flox; Alb-Cre mice (Fig. 8c and Supplementary Dataset 1). Among the 163 upregulated genes, 102 genes were found to be bound by Nrf2 in Nrf2 ChIP-seq data deposited in ENCODE and described in previous publications ${ }^{46,47}$ (Supplementary Dataset 2). Among the Nrf2 binding sites of these 102 genes, 51 genes contained an antioxidant response element, which is a consensus sequence for Nrf2 binding (Supplementary Dataset 2). This implies Nrf2 activation in livers of $H y D-L I R^{\text {flox/flox; }}$ Alb-Cre mice. Indeed, the gene expression of Nrf2-target genes, p62, Nqo1 and glutathione Stransferase, mu 1 (Gstm1) was induced in livers expressing HyDLIR-Venus, and the levels were similar to those in livers of Atgflox/ flox; Alb-Cre mice (Fig. 8d). We finally examined the phenotypes of $H y D-L I R^{\text {flox/flox; }}$ Alb-Cre mice, in which bulk autophagic activity is still intact. In contrast to severe liver enlargement in Atg 7 flox/flox; Alb-Cre mice, HyD-LIRflox/flox; Alb-Cre mice did not exhibit any hepatomegaly (Fig. 8e). Histological examination revealed that hepatocytes in HyD-LIR expressing livers were slightly larger, and possessed smaller glycogen regions compared to control liver, while Atg7-deficient hepatocytes had much larger eosinophilic cytoplasm than control or HyD-LIR expressing hepatocytes (Fig. 8f). The leakage of hepatic enzymes such as aspartate aminotransferase and alanine aminotransferase was evident in Atgflox/flox; Alb-Cre mice, but we did not observe any significant leakage in $H y D-L I R^{\text {flox/flox; }}$; Alb-Cre mice (Fig. 8g).

\section{Discussion}

Autophagosomes selectively surround several kinds of large protein complexes such as P-granule, stress granule, and Ape1 complex. The selective uptake of the complexes into autophagosomes is likely to be dependent on cargo liquidity. Actually, the aggregated Apel complex is not recognized as a selective substrate due to a failure of an adaptor protein to function on the aggregates $^{13}$. Once stress granules are affected by proteasome inhibition, which probably converts them into gel-like structures, they become targets for autophagy ${ }^{20}$. Likewise, when P-granules become less dynamic gel-like structures due to heat stress, they undergo autophagic degradation ${ }^{16}$. When 062 binds to ubiquitin chains, it acquires liquid-like properties ${ }^{17,18}$. Such phaseseparated droplets allow the exchange of their components, including ubiquitin and LC3, with the environment ${ }^{17}$. However, the properties of endogenous p62-structures remained unclear. In this study, we showed that endogenous p62-puncta are not typical liquid droplets, but gel-like structures (Fig. 1). Which factors change and/or keep the property of p62-gels? One hint is NBR1, a binding partner of p62. Since overexpression of NBR1 blocks the autophagic turnover of p62-structures ${ }^{19}$, the quantity of NBR1 bound to p62-structures might regulate the transition from a gellike structure to a liquid-droplet and in turn affect the direction of autophagic engulfment on the p62-gels. Since NBR1 is a stressinduced protein ${ }^{19}$, the transition might occur under stress conditions.

The formation of the autophagosome around phase-separated p62 requires sequential and antagonistic steps. First, a complex between p62 and FIP200 is formed to initiate the selective autophagy of $\mathrm{p} 62^{8}$. This complex is mutually exclusive to the one formed between p62 and LC3, indicating that the binding of p62 to LC3 occurs after dissociation from FIP $200^{8}$. We observed the formation of multiple isolation membranes/phagophores on the p62-gels (Fig. 2). Such feature is distinct from starvation induced autophagy ${ }^{48,49}$ and mitophagy ${ }^{50}$, in which single isolation membrane/phagophore is formed. In the case of mitophagy, a ubiquitin-binding adaptor protein NDP52 that is localized on damaged and then ubiquitinated mitochondria interacts with FIP200, leading to autophagosome formation around the mitochondria $^{10}$. Since NDP52 is unlikely to form liquid droplets, the 
a

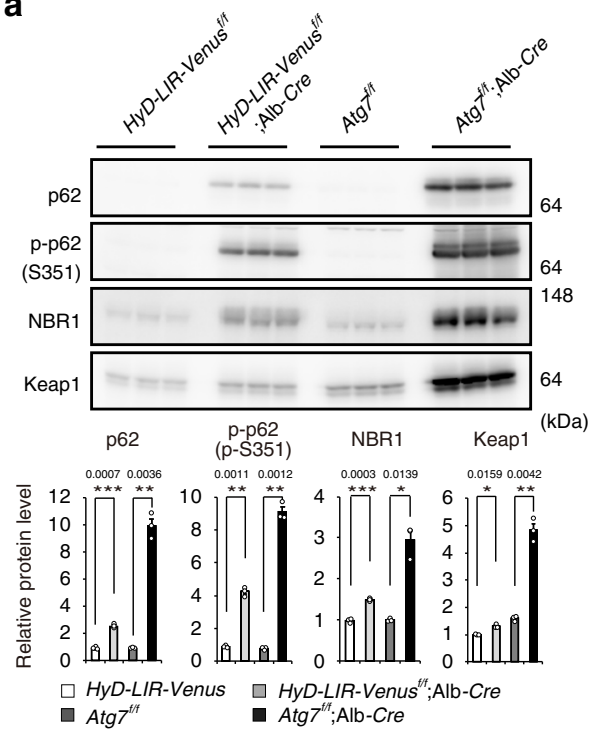

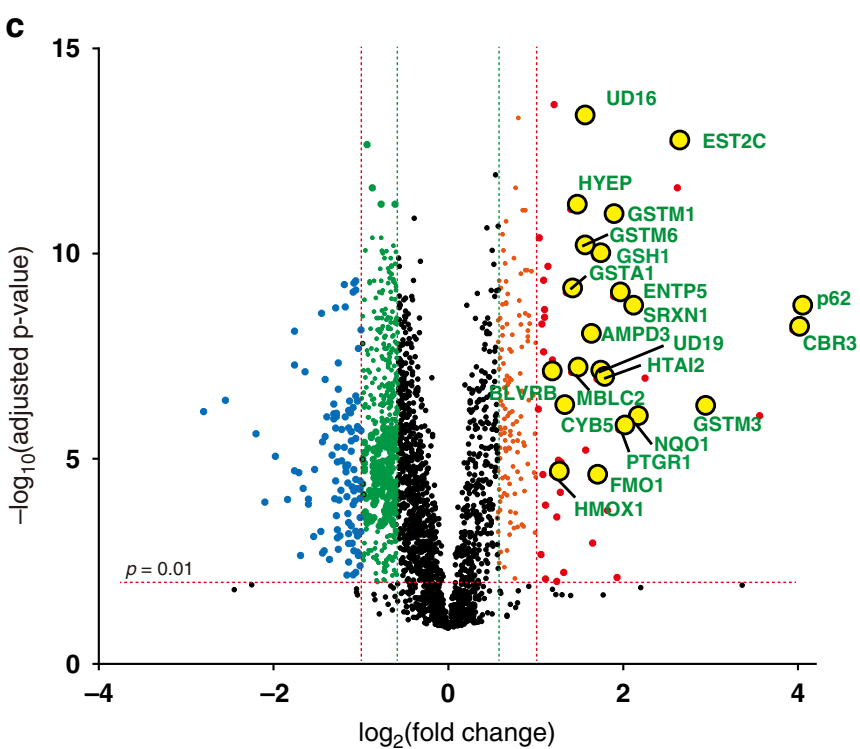

b
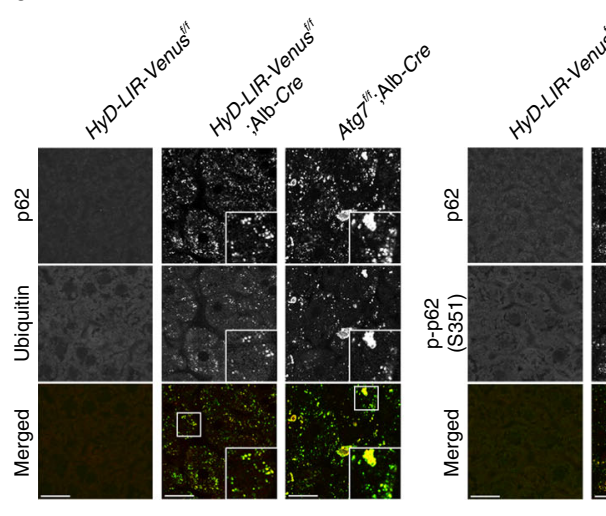
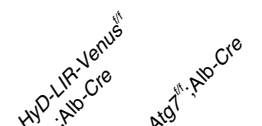

g

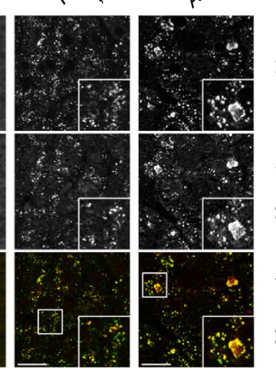

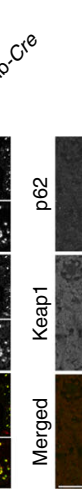

f

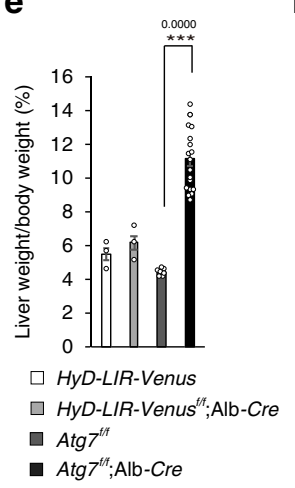

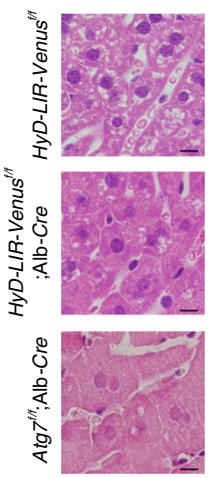

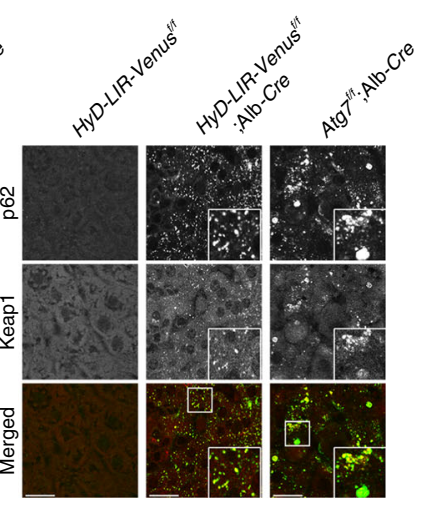

h

$\square$ HyD-LIR-Venus $\square$ HyD-LIR-Venus ; ; Alb-Cre

- $\operatorname{Atg}^{\text {tht }}$

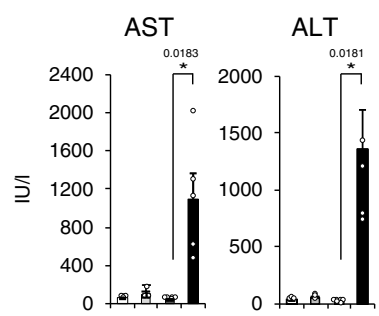

$\square$ HYD-LIR-Venus

$\square$ HyD-LIR-Venus ${ }^{t / 1}$;Alb-Cre

$\square \operatorname{Atg} 7^{t / t}$

- $\operatorname{Atg}^{7^{t /}}$;Alb-Cre d $\quad$ p62
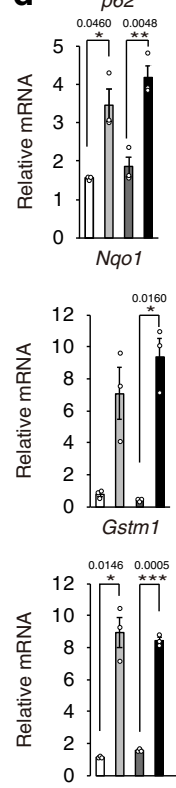

stoichiometry between NDP52 and FIP200 is likely to be lower compared with that between p62 and FIP200. Thus, it is plausible to suggest that the number of FIP200 on the cargo defines the number of isolation membranes/phagophores forming around the cargo. One p62-gel may interact with FIP200 molecules on multiple sites, which drives the formation of a number of isolation membranes/phagophores.

What is the role of LC3 in selective autophagy? It has been thought for a long time that the interaction of LC3 with selective substrates is indispensable to tether cargoes to elongating isolation membranes/phagophores. Considering selective autophagy in Saccharomyces cerevisiae ${ }^{51}$ as well as recent findings showing that ubiquitin-binding adaptors interact with FIP200 in mammalian cells ${ }^{8-10}$, isolation membrane/phagophore should be formed along the surface of selective substrates. While the blockade of the interaction of LC3 with selective substrates by overexpression of HyD-LIR did not affect autophagosome formation next to the p62-gels, isolation of p62-gels into the forming autophagosomes was almost completely cancelled (Fig. 4). Thus, the LC3-binding likely acts as driving force to direct isolation membranes/phagophore toward engulfment of the p62-gels. Similarly, Atg19, an adaptor protein for Ape1, and lipidated Atg8 are necessary and sufficient for selective sequestration of Ape1 droplets by membranes ${ }^{13}$. In sharp contrast, loss of LC3- and GABARAP-families in HeLa cells does not affect selective engulfment of damaged mitochondria by autophagosomes ${ }^{52}$. We 
Fig. 8 Physiological significance of p62-turnover. in vivo a Immunoblot analysis with homogenates from livers of three 5-week-old HyD-LIRflox/flox and HyD-LIR flox/flox; Alb-Cre mice. Data shown are representative of three separate experiments. Bar graphs indicate the quantitative densitometric analysis of the indicated proteins relative to whole proteins estimated by Ponceau-S staining. Data are means \pm s.e. ${ }^{\star} p<0.05$, ${ }^{\star *} p<0.01$, and ${ }^{\star \star \star} p<0.001$ as determined by two-sided Welch's $t$-test. Source data are provided as a Source Data file. $\mathbf{b}$ Immunohistofluorescence microscopy. Paraffin sections of mouse livers described in a were double-immunostained with p62 and ubiquitin, S351-phosphorylated p62 or Keap1 antibodies. Bars: 20 um. c Proteomics analysis with liver homogenates of mice described in $\mathbf{a}$. Nrf2-target genes are indicated in yellow color. $\mathbf{d}$ Gene expression of Nrf2-targets. Total RNAs were prepared from mouse livers of 5-week-old HyD-LIRflox/flox $(n=3)$ and HyD-LIRflox/flox; Alb-Cre mice $(n=3)$. Values were normalized against the amount of mRNA in HyD-LIR flox/flox livers. RT-qPCR analyses were performed as technical replicate on each biological sample. Data are means \pm s.e. ${ }^{\star} p<0.05,{ }^{\star \star} p<$ 0.01 , and ${ }^{* \star *} p<0.001$ as determined by two-sided Welch's $t$-test. Source data are provided as a Source Data file. e Liver weight (\% per body weight) of 5 week-old HyD-LIRflox/flox $(n=4), \operatorname{HyD}-$ LIR flox/flox; Alb-Cre $(n=4), \operatorname{Atg} 7$ flox/flox $(n=4)$, and Atg flox/flox; Alb-Cre $(n=4)$ mice. Data are means \pm s.e. ${ }^{\star \star \star} p<$ 0.001 as determined by two-sided Welch's $t$-test. Source data are provided as a Source Data file. $\mathbf{f}$ Hematoxylin and eosin staining of liver paraffin sections described in e. Bars: $50 \mu \mathrm{m}$. $\mathbf{g}$ Liver function tests of the mice used in 5-week-old HyD-LIR flox/flox $(n=4), \operatorname{HyD}-\mathrm{LIR}$ flox/flox; Alb-Cre $(n=4), A t g 7$ flox/flox $(n=$ 4), and Atgfflox/flox; Alb-Cre $(n=4)$ mice. The serum levels of aspartate aminotransferase (AST) and alanine aminotransferase (ALT) were measured. IU/ $\mathrm{L}$, international units/liter. Data are means \pm s.e. ${ }^{*} p<0.05$ as determined by two-sided Welch's $t$-test. Source data are provided as a Source Data file. g Schematic diagram of liver pathology.

observed slight but significant accumulation of mitochondria and prominent accumulation of ER in hepatocytes expressing HyDLIR (Supplementary Fig. S5). The importance of LC3-binding to substrates in selective autophagy may be dependent on substrates and cell types.

What is a biological role of the p62-gels? When the activity as an autophagy adaptor for ubiquitinated proteins is not required, p62 is held inactive through homodimerization of its ubiquitinassociated (UBA) domain, which prevents it from interacting with ubiquitin. Phosphorylation events drive the liberation of the UBA domain from dimeric inactivation; in particular, phosphorylation of serine 407 of p62 by ULK1 has been shown to facilitate the transition from dimer to monomer ${ }^{28}$. This modification is followed by phosphorylation of p62 on serine 403, either by ULK1, casein kinase 2 (CK2) or TANK-binding kinase 1 (TBK1), which enhances its binding to ubiquitin chains ${ }^{24,53,54}$. Once p62 binds to ubiquitin chains, the liquid-liquid phase separation occurs ${ }^{17,18}$. Besides a role of p62 in the condensation of ubiquitinated proteins, we propose that the p62-gel plays a central role in oxidative stress response, namely $\mathrm{Nrf2}$ activation. Keap1 was translocated onto the p62-gels, and its blockade suppressed the activation of Nrf2 (Figs. 5-7). The phosphorylation of p62 at Ser349, which is induced under selective autophagy conditions, enhances the interaction of p62 with Keap $1^{25}$. It is worth noting that this phosphorylation is also involved in the interaction of p62 with FIP $200^{8}$. These findings raise the possibility that once Ser349 of p62 is phosphorylated, selective autophagy and Nrf2 activation are concomitantly activated. Moreover, since the translocation of Keap1 onto the p62-gels was reversible (Fig. 6), the phosphorylation and/or de-phosphorylation of p62-Ser349 may participate in the regulation of both selective autophagy of p62-gels and oxidative stress response.

Due to marked accumulation of p62 and its phosphorylated forms in liver-specific autophagy-deficient mice (e.g. hepatocytespecific Atg5- and Atg7-knockout mice), Nrf2 is constitutively activated, which is believed to cause liver pathogenesis such as hepatomegaly, liver injury and development of adenoma ${ }^{12,35,39,55,56}$. However, these results were derived from phenotypic analyses of p62- or Nrf2-knockout mice under autophagy-deficient background. It was not unveiled whether Nrf2 activation, which is generally thought to be cytoprotective, is really cytotoxic or not. Utilizing HyD-LIR flox/flox; Alb-Cre mice, in which the turnover of p62-gels was compromised, we found that while liver Nrf2 is activated at similar extent to liver-specific Atg7-knockout liver, the mice did not show any pathological signs (Fig. 8). These results indicate that simple Nrf2 activation does not exert any cytotoxic effect on the liver and that the combination of Nrf2 activation with impaired autophagy causes liver injury. Consistently, liver-specific Keap1-knockout mice, in which Nrf2 is constantly activated without affecting autophagy, exhibit slight liver pathology ${ }^{57}$. Although we do not exclude the possibility that more severe liver phenotypes in Atg7knockout mice are derived from both defective autophagy and LC3associated phagocytosis (LAP), it was reported recently that LAPincompetent but autophagy-intact mice show no liver pathologies ${ }^{58}$. In conclusion, when both anabolism (Nrf2 activation) and catabolism (autophagy) are concomitantly deregulated, severe liver injury occurs (Fig. 8h).

In summary, our data indicate that endogenous p62 is present as gel-like structures, the p62-gels serve as platforms for autophagosome formation and for Nrf2 activation, and that the p62mediated Nrf2 activation itself is not cytotoxic.

\section{Methods}

Cell culture. Huh-1 (JCRB0199), MEFs, and HeLa (ATCC CCL-2) cells were grown in Dulbecco's modified Eagle medium (DMEM) containing 10\% fetal bovine serum (FBS), $5 \mathrm{U} / \mathrm{ml}$ penicillin, and $50 \mu \mathrm{g} / \mathrm{ml}$ streptomycin. For overexpression experiments, Huh-1 cells were transfected with Lipofectamine 3000 (Thermo Fisher Scientific, Waltham, MA, USA). p62-GFPKI/+ and Atg $7^{-/-}$; p62-GFPKI/+ MEFs were used in this study. Huh-1 and HeLa cells were authenticated by STR profile. All cell lines were tested for mycoplasma contamination.

Mice. For the generation of Rosa26-loxP-stop-loxP-HyD-LIR-Venus mice (HyD LIR-Venus mice), a genomic fragment that contained the ROSA locus was isolated from C57BL/6 mouse genomic BAC clone from an RP23 mouse genomic BAC library (Advanced Geno TEchs Co). The targeting vector had a splice acceptor (SA), two loxP sequences that were inserted before the repeated SV40 polyadenylation signal and after the "stop" sequence that contained the terminator of the yeast His3 gene and SV40 polyadenylation signal. A 1.7-kb fragment of the FRT-PGK-gb2-neo-FRT-loxP cassette (Gene Bridges) was inserted after the repeated SV40 polyadenylation signal. After the second loxP sequence, the targeting vector contained the coding sequence of the DTR, simian HBEGF followed by IRES and tdTomato sequences. The targeting vectors were electroporated into mouse RENKA ES cells, selected with G418 (250 $\mu \mathrm{g} / \mathrm{ml}$; Invitrogen, San Diego, CA), and then screened for homologous recombinants by Southern blot analysis. Southern blot analysis in $H y D-L I R-V e n u s$ mice was performed by digesting genomic DNA with NdeI (Takara Bio, Inc., Shiga, Japan) or EcoRV (Takara Bio, Inc.), followed by hybridization to detect wild-type $12.7-\mathrm{kb}$ and flox $9.7-\mathrm{kb}$ bands or wild-type 11.5 $\mathrm{kb}$ and flox 19.3-kb bands. Mice were housed in specific pathogen-free facilities, and the Ethics Review Committee for Animal Experimentation of Juntendo University approved the experimental protocol. We have complied with all relevant ethical regulations.

RT-qPCR (real-time quantitative reverse transcriptase PCR). Using the Transcriptor First-Strand cDNA Synthesis Kit (Roche Applied Science, Indianapolis, IN, USA), cDNA was synthesized from $1 \mu \mathrm{g}$ of total RNA. RT-qPCR was performed using the LightCycler ${ }^{\circledast} 480$ Probes Master mix (Roche Applied Science) on a LightCycler ${ }^{\circledast} 480$ with LightCycler $^{\circledR} 480$ Software version 1.5.1 (Roche Applied Science). Signals from human and mouse samples were normalized against GAPDH (glyceraldehyde-3-phosphate dehydrogenase) and Gusb (B-glucuronidase) mRNA, respectively. The sequences of primers used for gene expression analysis in either mouse livers or human cell lines are provided in Supplementary Table S1. 
Immunoblot analysis. Livers were homogenized in $0.25 \mathrm{M}$ sucrose, $10 \mathrm{mM}$ 2-[4(2-hydroxyethyl)-1-piperazinyl] ethanesulfonic acid (HEPES) (pH 7.4), and $1 \mathrm{mM}$ dithiothreitol (DTT). Cells were lysed in ice-cold TNE buffer (50 mM Tris- $\mathrm{HCl}, \mathrm{pH}$ 7.5, $150 \mathrm{mM} \mathrm{NaCl}, 1 \mathrm{mM}$ EDTA) containing 1\% SDS and protease inhibitors. Nuclear and cytoplasmic fractions from cultured cells were prepared using the NEPER Nuclear and Cytoplasmic Extraction Reagents (Thermo Fisher Scientific). For immunoprecipitation analysis, Huh-1 cells were grown on six-well plates and treated with KMN003 for $12 \mathrm{~h}$ at the indicated concentrations. The cells were lysed in $300 \mu \mathrm{l}$ of IP buffer ( $20 \mathrm{mM}$ Tris- $\mathrm{HCl}$ [pH 7.5], $150 \mathrm{mM} \mathrm{NaCl}, 1 \mathrm{mM}$ EDTA, $1 \%$ NP40, 1\%TX-100) containing Protease inhibitor cocktail (Roche), and the lysates were then centrifuged at $20,000 \times g$ for $10 \mathrm{~min}$ at $4{ }^{\circ} \mathrm{C}$ to remove debris. In the next step, $200 \mu \mathrm{l}$ of IP buffer, $0.5 \mu \mathrm{l}$ of anti-Keap1 antibody (10503-2-AP, PGI) and $10 \mu \mathrm{l}$ of Protein G Sepharose 4 Fast Flow (Cytiva) were added to the $200 \mu \mathrm{l}$ of lysate, and the mixture was mixed under constant rotation for $3 \mathrm{~h}$ at $4{ }^{\circ} \mathrm{C}$. The immunoprecipitates were washed three times with ice-cold IP buffer. The complex was boiled for $5 \mathrm{~min}$ in SDS sample buffer in the presence of $\beta$-Mercaptoethanol to elute proteins. For pull-down assay, HEK293T cells were transfected with One-StrepFLAG-tagged p62 (OSF-p62). Twenty-four hour after the transfection, the cell lysate was subjected to StepTactin Sepharose (Cytiva), and then purified Keap1-DC (321-609aa) was added to the StepTactin Sepharose-conjugated OSF-p62. The complex consisting of Keap1-DC and OSF-p62 was treated in presence or absence of the KMN003 in pull-down assay buffer ( $20 \mathrm{mM}$ Tris- $\mathrm{HCl}$ [pH 7.5], $150 \mathrm{mM}$ $\mathrm{NaCl}, 0.5 \% \mathrm{NP} 40,0.5 \mathrm{mM}$ TCEP). The pulled-down protein complexes were washed with pull-down assay buffer and then boiled for 5 min in SDS sample buffer in the presence of $\beta$-Mercaptoethanol to elute proteins. Samples were subjected to SDS-PAGE, and transferred to a polyvinylidene difluoride membrane thereafter (Merck, IPVH00010). Antibodies against p62 (GP62-C, Progen Biotechnik GmbH, Heidelberg, Germany; 1:1000), S403-phosphorylated p62

(GTX128171, GeneTex, CA, USA; 1:1000), NBR1 (4BR; Santa Cruz Biotechnology, Dallas, TX USA; 1:1000), LC3B (\#2775, Cell Signaling Technology; 1:500), KEAP1 (10503-2-AP, proteintech, Rosemont, USA; 1:1000), Nqo1 (ab34173; Abcam, Cambridge, UK; 1:2000), Gclc (ab41463, Abcam; 1:500), GSTm1 (GSTM12-S, ALPHA DIAGNOSTIC INTERNATIONAL, San Antonio, USA; 1:1000), and GFP (594, Medical and Biological Laboratories, Nagoya, Japan; 1:1000) were purchased from the indicated suppliers. Anti-S349-phosphorylated p62 polyclonal antibody $(1: 1000)$ was raised in rabbits by using the peptide Cys+KEVDP(pS)TGELQSL as an antigen. Blots were incubated with horseradish peroxidase-conjugated goat antimouse IgG $(\mathrm{H}+\mathrm{L})$ (Jackson ImmunoResearch Laboratories, Inc., 115-035-166), goat anti-rabbit IgG $(\mathrm{H}+\mathrm{L})$ (Jackson ImmunoResearch Laboratories, Inc., 111035-144; $1: 10000)$ or goat anti-guinea pig $\operatorname{IgG}(\mathrm{H}+\mathrm{L})$ antibody (Jackson ImmunoResearch Laboratories, Inc., 106-035-003; 1:10000), and visualized by chemiluminescence. Band density was measured using the software Multi Gauge V3.2 (FUJIFILM Corporation, Tokyo, Japan). Uncropped and unprocessed scans of the all immunoblots were supplied in the Source Data file.

Immunofluorescence microscopy. Cells grown on coverslips were fixed in $4 \%$ paraformaldehyde in PBS for 10 min, permeabilized with $0.1 \%$ Triton X-100 or 50 $\mathrm{ng} / \mathrm{ml}$ Digitonin in PBS for $5 \mathrm{~min}$, blocked with $0.1 \%$ (w/v) gelatin (Sigma-Aldrich) in PBS for $45 \mathrm{~min}$, and then incubated overnight with primary antibodies diluted 1:200 in gelatin/PBS. After washing, cells were incubated with Goat anti-Guinea pig IgG $(\mathrm{H}+\mathrm{L})$ Cross-Adsorbed Secondary Antibody, Alexa Fluor 488 (A11073, Thermo Fisher Scientific), Goat anti-Mouse IgG $(\mathrm{H}+\mathrm{L})$ Highly Cross-Adsorbed Secondary Antibody, Alexa Fluor 647 (A21236, Thermo Fisher Scientific) or Goat anti-Rabbit IgG $(\mathrm{H}+\mathrm{L})$ Highly Cross-Adsorbed Secondary Antibody, Alexa Fluor 647 (A21245, Thermo Fisher Scientific) at a dilution ratio of 1:1000 for $60 \mathrm{~min}$. For treatment of 1,6-Hexandiol, GFP-p62 was transfected in Huh-1 cells. twnty-four hour after transfection, the cells were lysed in ice-cold lysis buffer $(50 \mathrm{mM}$ Tris$\mathrm{HCl}, \mathrm{pH} 7.4,150 \mathrm{mM} \mathrm{NaCl}, 1 \mathrm{mM}$ EDTA) containing $0.2 \%$ Triton X-100 and 65 $\mathrm{U} / \mathrm{mL}$ RNasin ${ }^{\circledR}$ Ribonuclease Inhibitors (Promega). The lysates were further passed through 27 -gauge needle 20 times and incubated for $20 \mathrm{~min}$ at $4{ }^{\circ} \mathrm{C}$. The resulting lysates were centrifuged at $200 \times g$ for $5 \mathrm{~min}$. The supernatant was mixed with 10 $\mathrm{mM} \mathrm{MgSO} 4,1 \mathrm{mM} \mathrm{CaCl} 2$ and $4 \mathrm{U} / \mathrm{mLRQ} 1 \mathrm{RNase}$ Free DNase (Promega) and incubated for $30 \mathrm{~min}$ at RT. The mixture was centrifuged at $10,000 \times \mathrm{g}$ for $7 \mathrm{~min}$. The pellet containing GFP-p62 labelled droplets was mixed with 1,6-Hexandiol (final concentration 10\%). Cells and droplets were imaged using a confocal laserscanning microscope FV1000 with FV10-ASW 04.01 or FV3000 with FV31S-SW 2.4.1.198 (Olympus) with a UPlanSApo $\times 60$ NA 1.40 oil objective lens. To measure the fluorescence recovery after photobleaching (FRAP), cells were grown in $35 \mathrm{~mm}$ glass base dishes (Iwaki, Japan). p62-bodies were bleached for $3 \mathrm{sec}$ using a laser intensity of $70 \%$ at $480 \mathrm{~nm}$, and then the recovery was recorded for the indicated time. After image acquisition, contrast and brightness were adjusted using Photoshop 2021v25.0 (Adobe).

Correlative light and electron microscopy (CLEM). Detailed CLEM method was recently published ${ }^{59}$. Briefly, p62-GFPKI/+ MEFs were cultured on coverslips etched with $150-\mu \mathrm{m}$ grids (Matsunami Glass Ind., Ltd.). The MEFs were treated by sodium arsenite (As[III]) for $10 \mathrm{hr}$ and then cultured in regular medium for $3 \mathrm{~h}$. They were fixed with $2 \%$ PA- $0.5 \%$ glutaraldehyde (GA) in $0.1 \mathrm{M} \mathrm{PB} \mathrm{(pH} \mathrm{7.4),} \mathrm{and}$ phase-contrast and fluorescence images were obtained using a confocal microscope (FV1000; Olympus). The cells were fixed again with 2\% PA and 2\% GA in $0.1 \mathrm{M}$
$\mathrm{PB}$ ( $\mathrm{pH}$ 7.4), processed using the reduced-osmium method, and embedded in Epon812. Areas containing cells of interest were trimmed, cut as serial $70-\mathrm{nm}$ or 200-nm sections, and observed with an electron microscope (EM; JEM1400 Plus; JEOL). Alignment of light microscopy and EM images was performed using TrakEM2 software version 1.0a $2012-07-04^{60}$ (https://imagej.net/TrakEM2).

Tomography. Electron tomography was performed on 200-nm serial sections using 15-nm gold particles deposited on both surfaces of the grids as fiducial markers. JEM1400 Plus operated at $120 \mathrm{kV}$ was used to collect dual-axis tilt series from $-65^{\circ}$ to $+65^{\circ}$ with $1.5^{\circ}$ steps using SerialEM version $3.3 .1^{61}$. Tomograms were generated using the IMOD software package version $4.7 .3^{62}$. The tomograms where segmented in Microscopy Image Browser (version 2.651/25.04.2020) ${ }^{63}$ and then exported to 3D Slicer software (https://www.slicer.org/) ${ }^{64}$ in order refine the models and make the final images. ImageJ (the core package in Fiji) version Image) $1.53 \mathrm{c}$ (https://imagej.net/Fiji) ${ }^{65}$ was used for general image handling. Fig. 2 and Supplementary Fig. S2 were created using Corel draw version 19.0.0.328 (https:// www.coreldraw.com/en/).

\section{Electron microscopy (EM) and immuno-EM for primary hepatocytes. For} conventional EM, primary hepatocytes were fixed in $0.1 \mathrm{M} \mathrm{PB}$ containing $2 \%$ paraformaldehyde and $2 \%$ glutaraldehyde, followed by a process of the reducedosmium method $^{66}$, embedding in Epon812, sectioning, and staining with uranyl acetate and lead citrate. For immune-EM, the cells were fixed in $0.1 \mathrm{M}$ PB containing $4 \%$ paraformaldehyde and $4 \%$ sucrose, and then frozen after infusion with $2.3 \mathrm{M}$ sucrose and $20 \%$ polyvinylpyrrolidone ${ }^{67}$. Ultrathin sections were mounted on Formvar carbon-coated nickel grids, blocked with $1 \%$ bovine serum albumin (BSA) in PBS, incubated with anti-p62 (GP62-C, Progen) antibody, and then incubated with colloidal gold $(6 \mathrm{~nm})$-conjugated goat anti-guinea pig antibody (Jackson ImmunoResearch laboratories, Inc). The sections were observed with an electron microscope (EM; JEM1400EX; JEOL)

Immunohistofluorescence microscopy. Mouse livers were quickly excised, cut into small pieces, and then fixed by immersing in $4 \%$ paraformaldehyde- $4 \%$ sucrose in $0.1 \mathrm{M}$ phosphate buffer, $\mathrm{pH} 7.4(\mathrm{~PB})$. After rinsing, they were embedded in paraffin or OCT compound for hematoxylin-eosin staining and immunostaining. Paraffin- and cryosections were prepared and processed for antigen retrieval for $20 \mathrm{~min}$ at $98^{\circ} \mathrm{C}$ using a microwave processor (MI-77, AZUMAYA, Japan) in $1 \%$ immunosaver (Nissin EM, Japan). Sections were blocked and incubated for 2-3 days at $4{ }^{\circ} \mathrm{C}$ with the following first antibodies: guinea pig polyclonal antibody against p62 (GP62-C, Progen), rabbit polyclonal antibody against phosphorylated p62 $2^{25}$ or ubiquitin (Z0458, Dako), and mouse monoclonal antibody against Keap1 (Proteintech Group). They were further incubated Alexa647-conjugated donkey anti-guinea pig IgG (706-605-148, Jackson ImmunoResearch laboratories, Inc., West Grove, PA), and Alexa594-conjugated donkey anti-rabbit IgG (711-585-152, Jackson ImmunoResearch laboratories, Inc.) or Alexa594-conjugated donkey antimouse IgG (715-585-150, Jackson ImmunoResearch laboratories, Inc.). HE-images were acquired with a microscope (BX51, Olympus, Japan) equipped with a cooled CCD camera system (DP-71, Olympus), and immunofluorescence images were taken by a laser-scanning confocal microscope (FV1000, Olympus) with 60x objective lens (PlanApo N 60x, NA 1.42 oil). After image acquisition, contrast and brightness were adjusted using Photoshop CS6 (Adobe).

Plasmids for recombinant protein expression in bacteria. The pGEX6P-1 vector (GE Healthcare) was selected for production of N-terminal glutathione Stransferase (GST) fusion proteins in E. coli cells. For construction of pGEX6PmCherry-p62, the gene encoding mCherry-p62 was amplified by PCR and inserted into the downstream region of HRV $3 \mathrm{C}$ protease recognition site. The gene encoding linear tetraubiquitin $(4 \mathrm{xUb})$ was purchased from GENEWIZ and inserted into the downstream region of HRV $3 \mathrm{C}$ protease recognition site of the pGEX6P-1 vector. For construction of pGEX6P-SNAP-4xUb, the gene coding SNAP-tag was inserted into the upstream region of $4 \mathrm{xUb}$ of pGEX6P-4xUb. For construction of pGEX6P-SNAP-Keap1, the gene coding Keap1 with an N-terminal SNAP-tag was inserted into the downstream region of the pGEX6P-1 vector. All gene insertions were performed by NEBuilder HiFi DNA Assembly (New England Biolabs). Mutations to generate the indicated amino acid substitutions were introduced by PCR-mediated site-directed mutagenesis. All constructs were sequenced to confirm accuracy of cloning.

Protein expression and purification. E. coli strain BL21(DE3) cells were used for expression of all recombinant proteins. Protein expression was induced by $0.1 \mathrm{mM}$ isopropyl $\beta$-D-thiogalactopyranoside (IPTG) at $16-18^{\circ} \mathrm{C}$ overnight. The E. coli cells were harvested by centrifugation and lysed by sonication in Buffer A $(50 \mathrm{mM}$ HEPES- $\mathrm{NaOH}$ pH 8.0, $500 \mathrm{mM} \mathrm{NaCl}$ ) supplemented with $1 \mathrm{mM}$ Tris(2-carboxyethyl)phosphine (TCEP), $1 \mathrm{mM}$ phenylmethylsulfonyl fluoride, and $1 \mathrm{x}$ protease inhibitor cocktail (EDTA free) (Nacalai tesque). For the E. coli cells expressing GST-SNAP-Keap1, DNase I (Takara) and RNase A (Nacalai tesque) were added to Buffer A. After removal of insoluble cell debris by centrifugation, the supernatant was applied to GST accept resin (Nacalai tesque). After washing the resin with Buffer A, HRV 3C protease with a GST-tag was added to the resin. After the on- 
column cleavage for $\sim 16 \mathrm{~h}$ at $4{ }^{\circ} \mathrm{C}$, the cleaved proteins were collected and concentrated to at least $50 \mu \mathrm{M}$ for mCherry-p62 WT, mCherry-p62 W338A L341A, and SNAP-Keap1 or at least $500 \mu \mathrm{M}$ for SNAP-4xUb and 4xUb in Buffer A supplemented with $10 \%$ glycerol using Vivaspin 500 (GE Healthcare). SNAP-4xUb and SNAP-Keap1 were labelled with SNAP-Surface Alexa Fluor 488 and SNAPSurface Alexa Fluor 647 (New England Biolabs), respectively. mKalama1Atg8K26P and Atg8 conjugation enzymes (Atg3, Atg7, Atg16, and Atg5-Atg12 conjugate) were prepared as previously reported ${ }^{13}$. Purified proteins were stored at $-80^{\circ} \mathrm{C}$ until use.

Preparation Atg8-GUVs. GUVs were prepared as previously reported ${ }^{13}$. Briefly, $100 \mu \mathrm{L}$ of a $1 \mathrm{mM}$ phospholipid mixture consisting of 1-palmitoyl-2-oleoyl-snglycero-3-phosphocholine (POPC), 1-palmitoyl-2-oleoyl-sn-glycero-3-phosphoethanolamine (POPE), L- $\alpha$-phosphatidylinositol (Liver, PI) (all from Avanti Polar Lipids) at a molar ratio of 5:4:1 in chloroform was prepared in a 5-mL glass vial and dried under a gentle stream of nitrogen to produce a thin, homogeneous lipid film. For GUVs labelled with Cy5, $0.01 \mathrm{mM}$ 1,2-dioleoyl-sn-glycero-3-phosphoethanolamine-N-(Cyanine 5) (Cy5-DOPE) (Avanti Polar Lipids) was added to $1 \mathrm{mM}$ phospholipid mixture. The glass vial was put in a vacuum desiccator overnight. After thin lipid film was prehydrated with $20 \mu \mathrm{L}$ of MilliQ water at $60^{\circ} \mathrm{C}$ for $7 \mathrm{~min}, 500 \mu \mathrm{L}$ of Buffer B (20 mM HEPES pH 7.5, $150 \mathrm{mM} \mathrm{NaCl}, 0.1 \mathrm{M}$ sucrose) was added and incubated for $2-3 \mathrm{hr}$ at $60^{\circ} \mathrm{C}$. The solution containing GUVs was transferred to $1.5-\mathrm{mL}$ microcentrifuge tube and centrifuged at $13,000 \times$ $g$ for $30 \mathrm{~min}$ at $25^{\circ} \mathrm{C}$. After removal of precipitates, the GUV solution was diluted with Buffer C (20 mM HEPES pH 7.5, $150 \mathrm{mM} \mathrm{NaCl}, 0.1 \mathrm{M}$ glucose). For the lipidation of Atg8 on the GUV membrane (Atg8-GUV), a $10 \mu \mathrm{L}$ mixture of $1 \mu \mathrm{M}$ mKalama1-Atg8K26P, 0.5 $\mu \mathrm{M}$ Atg3, $0.5 \mu \mathrm{M}$ Atg7, 0.25 $\mu \mathrm{M}$ Atg12-Atg5-Atg16 in Buffer C supplemented with $1 \mathrm{mM}$ ATP and $1 \mathrm{mM} \mathrm{MgCl}_{2}$ was added to a $10 \mu \mathrm{L}$ GUV solution and incubated for $1 \mathrm{~h}$ at $\sim 23^{\circ} \mathrm{C}$.

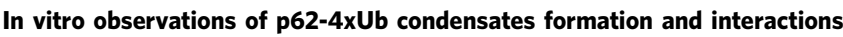
between Atg8-GUV and p62-4xUb condensates. Fluorescence observation was performed on glass-bottom dishes (MatTek) coated with $0.3 \%$ (w/v) bovine serum albumin in Buffer $\mathrm{C}$ using an FV3000RS confocal laser-scanning microscope (Olympus). 405, 488, 561, and 640-nm lasers were used for excitation of mKalama1, Alexa Fluor 488, mCherry and Alexa Fluor 647 or Cy5, respectively. To observe p62-4xUb condensates, $5 \mu \mathrm{M}$ mCherry-p62 or mCherry-p62 W338A L341A was mixed with $50 \mu \mathrm{M} 4 \mathrm{xUb}$ and $5 \mu \mathrm{M}$ labelled SNAP-4xUb dissolved in 20 $\mu \mathrm{L}$ of Buffer D (20 mM HEPES-NaOH pH 7.5, $150 \mathrm{mM} \mathrm{NaCl}, 1 \mathrm{mM}$ TCEP). To observe p62-4xUb-Keap1 condensates, $5 \mu \mathrm{M}$ mCherry-p62 was mixed with $50 \mu \mathrm{M}$ $4 \mathrm{xUb}, 5 \mu \mathrm{M}$ labelled SNAP-4xUb and $5 \mu \mathrm{M}$ labelled SNAP-Keap1 dissolved in 20 $\mu \mathrm{L}$ of Buffer D. To observe the interactions between Atg8-GUV (or Atg8-GUV labelled with Cy5) and p62-4xUb condensates, $5 \mu \mathrm{M}$ mCherry-p62 or mCherryp62 W338A L341A was mixed with $20 \mu \mathrm{L}$ Atg8-GUV solution containing $50 \mu \mathrm{M}$ $4 \mathrm{xUb}$ and $5 \mu \mathrm{M}$ labelled SNAP-4xUb. To observe the interactions between Atg8GUV and p62-4xUb-Keap1 condensates, $5 \mu \mathrm{M}$ mCherry-p62 was mixed with $20 \mu \mathrm{L}$ Atg8-GUV solution containing $50 \mu \mathrm{M} 4 \mathrm{xUb}, 5 \mu \mathrm{M}$ labelled SNAP-4xUb and $5 \mu \mathrm{M}$ labelled SNAP-Keap1. Each mixed solution was incubated for $1 \mathrm{~h}$ at $\sim 23^{\circ} \mathrm{C}$ before observation.

Mass spectrometry analysis. Mass spectrometry analyses were performed on a TripleTOF 5600 mass spectrometer coupled to Ekspert nanoLC 415 system with Ekspert cHipLC (Sciex, Framingham, MA). The samples were injected onto a Trap column of $200 \mu \mathrm{m} \times 0.5 \mathrm{~mm}$ ChromXP C18-CL, $120 \AA$ pore size, $3 \mu \mathrm{m}$ diameter particles and $75 \mu \mathrm{m} \times 150 \mathrm{~mm}$ ChromXP C18-CL analysis column (Sciex). Samples were run with a $100 \mathrm{~min}$ gradient from $2-40 \%$ solvent B (solvent A: $0.1 \%$ formic acid, solvent B: $0.1 \%$ formic acid, $80 \%$ acetonitrile) at a flow rate of $300 \mathrm{nl} / \mathrm{min}$. The analysis results were searched against the UniProt database (release 2019_11) using ProteinPilot 5.0.2. software (Sciex). The library contained 2619 protein groups with the cutoff of $1 \%$ false discovery rate. Similar LC gradient and mass spectrometer settings as for the IDA acquisition described above were used, but the mass spectrometer was operated in SWATH mode. The precursor isolation windows overlap by $1 \mathrm{~m} / \mathrm{z}$ and cover a range of $400-1250 \mathrm{~m} / \mathrm{z}$. The variable isolation windows were determined with SWATH variable window calculator tool version 1.0 (Sciex). The MS2 spectra were recorded with an accumulation time of $40 \mathrm{~ms}$ and cover $100-1600 \mathrm{~m} / z$. PeakView 2.2 (Sciex) was used to analyze the SWATH data. Proteins with one to five peptides were used for quantitative analysis. The sum of peptide peak area was used for protein quantification.

Unpaired student's $t$-tests were performed to compare the SWATH results for the HyD-LIR-Venus expressing liver groups and control groups. FDR corrections for the $P$ values were carried out with Storey's method ${ }^{68,69}$. The up- and downregulated proteins with the adjusted $P$ values less than 0.01 were used for the following the DAVID enrichment analysis ${ }^{70}$. The DAVID analysis was performed to obtain detailed information on biological functions and pathways that significantly enriched up- and downregulated proteins in SWATH analysis.

Analysis of ChIP-seq dataset for NRF2 binding. NRF2 ChIP-seq data in MEL and $\mathrm{CH} 12$ cells were obtained from ENCODE database via Integrative Genomics Viewer (IGV 2.3.65). Those in diethylmaleate-treated Hepalc1c7 cells and Keap1- deficient mouse bone-marrow-derived macrophages were obtained from previous publications ${ }^{46,47}$. If the submit of NRF2 binding peaks were contained within the gene body and flanking intergenic regions in at least one of the four NRF2 ChIPseq data sets, the gene was considered to have NRF2 binding. The NRF2 binding regions were searched for the antioxidant response element $(\mathrm{GCnnn} \mathrm{G} / \mathrm{CTCA} / \mathrm{C})$, which is a consensus sequence of NRF2 binding.

Protein degradation assay. Hepatocytes were plated at $5 \times 10^{4}$ cells/well in collagen-coated 24-well plates and cultured in Williams'E medium with $10 \%$ FCS (Williams E/10 \% FCS) for $24 \mathrm{hr}$. Cells were incubated with Williams E/10 \% FCS containing $\left[{ }^{14} \mathrm{C}\right]$-leucine $(0.5 \mu \mathrm{Ci} / \mathrm{ml})$ for $24 \mathrm{~h}$ to label long-lived proteins. Cells were washed with Williams E/10 \% FCS containing $2 \mathrm{mM}$ unlabelled leucine and incubated with the medium for $2 \mathrm{~h}$ to allow degradation of short-lived proteins and minimize the incorporation of labeled leucine, released by proteolysis into protein. The cells were then washed with PBS, and incubated at $37^{\circ} \mathrm{C}$ with Krebs-Ringer bicarbonate (KRB) medium and Williams $\mathrm{E} / 10 \% \mathrm{FCS}$ in the presence or absence of protease inhibitors [ $5 \mathrm{mM}$ monomethylamine (MA), $10 \mu \mathrm{g} / \mathrm{mL}$ E64d and pepstatin $(\mathrm{E} / \mathrm{P})$ or $5 \mu \mathrm{M}$ epoxomicin]. After $4 \mathrm{~h}$, aliquots of the medium were taken and a one-tenth volume of $100 \%$ trichloroacetic acid was added to each aliquot. The mixtures were centrifuged at $12,000 \times g$ for $5 \mathrm{~min}$, and the acid-soluble radioactivity was determined using a liquid scintillation counter. At the end of the experiment, the cultures were washed twice with PBS, and $1 \mathrm{ml}$ of cold trichloroacetic acid was added to fix the cell proteins. The fixed cell monolayers were washed with trichloroacetic acid and dissolved in $1 \mathrm{ml}$ of $1 \mathrm{~N} \mathrm{NaOH}$ at $37^{\circ} \mathrm{C}$. Radioactivity in an aliquot of $1 \mathrm{~N} \mathrm{NaOH}$ was determined by liquid scintillation counting. The percent protein degradation was calculated according to the published procedure.

Purification of p62-gels. To purify p62-gels from Huh-1 cells, we modified and used a method for purification of P-body in cells that was developed by elsewhere ${ }^{71}$. Briefly, GFP-tagged p62 was expressed into Huh-1 cells. Twenty-four hour after transfection, the cells were suspended with ice-cold lysis buffer $(50 \mathrm{mM}$ Tris-HCl, pH 7.4, $150 \mathrm{mM} \mathrm{NaCl}, 1 \mathrm{mM}$ EDTA) containing $65 \mathrm{U} / \mathrm{mL}$ RNasin ${ }^{\circledast}$ Ribonuclease Inhibitors (Promega) and EDTA-free Protease inhibitor cocktail (Roche) and then lysed by passing 20 times through a $27 \mathrm{Gx} 3 / 4$. The lysates were incubated on ice for $20 \mathrm{~min}$ and then centrifuged at $200 \times \mathrm{g}$ for $5 \mathrm{~min}$ at $4{ }^{\circ} \mathrm{C}$ to delete nuclei. The resultant supernatants were supplemented with $10 \mathrm{mM} \mathrm{MgSO}_{4}$ and $1 \mathrm{mM} \mathrm{CaCl} 2$ and treated with $4 \mathrm{U} / \mathrm{mL}$ of RQ1 DNase (Promega) for $30 \mathrm{~min}$ at RT. Thereafter, the mixtures were spun at $10,000 \times g$ for $7 \mathrm{~min}$ at $4{ }^{\circ} \mathrm{C}$, and pellets were resuspended into $10 \mathrm{~mL}$ of lysis buffer with 80 Units of RNaseOut (Promega). From this fraction, p62-bodies were sorted on BD FACSAria ${ }^{\text {mit }}$ II Cell Sorter with BD FACSDiva software v8.0 (BD BioSciences). Particles were detected according to their Forward-scattered light (FSC) and their green fluorescence using the $488 \mathrm{~nm}$ excitation laser and the 526/52 band pass filter.

Statistics and reproducibility. Values, including those displayed in the graphs, are means \pm s.e. Statistical analysis was performed by the unpaired $t$-test (Welch test) with Microsoft Excel 2011 (Microsoft). A $P$ value less than 0.05 was considered to indicate statistical significance. Each experiment was repeated independently at least three times with similar results.

Reporting summary. Further information on research design is available in the Nature Research Reporting Summary linked to this article.

\section{Data availability}

The accession numbers are PXD019492 for ProteomeXchange and JPST000830 or jPOST. All figures and movies are available in figshare (https://doi.org $/ 10.6084 / \mathrm{m} 9$. figshare.13218746). Source data are provided with this paper.

Received: 18 May 2020; Accepted: 17 November 2020; Published online: 04 January 2021

\section{References}

1. Ohsumi, Y. Historical landmarks of autophagy research. Cell Res. 24, 9-23 (2014).

2. Dikic, I. \& Elazar, Z. Mechanism and medical implications of mammalian autophagy. Nat. Rev. Mol. Cell Biol. 19, 349-364 (2018).

3. Lamb, C. A., Yoshimori, T. \& Tooze, S. A. The autophagosome: origins unknown, biogenesis complex. Nat. Rev. Mol. Cell Biol. 14, 759-774 (2013).

4. Gatica, D., Lahiri, V. \& Klionsky, D. J. Cargo recognition and degradation by selective autophagy. Nat. Cell Biol. 20, 233-242 (2018).

5. Kaur, J. \& Debnath, J. Autophagy at the crossroads of catabolism and anabolism. Nat. Rev. Mol. Cell Biol. 16, 461-472 (2015).

6. Mizushima, N. \& Komatsu, M. Autophagy: renovation of cells and tissues. Cell 147, 728-741 (2011). 
7. Khaminets, A., Behl, C. \& Dikic, I. Ubiquitin-dependent and independent signals in selective autophagy. Trends Cell Biol. 26, 6-16 (2016).

8. Turco, E. et al. FIP200 claw domain binding to $\mathrm{p} 62$ promotes autophagosome formation at ubiquitin condensates. Mol. Cell 74, 330-346 e311 (2019).

9. Ravenhill, B. J. et al. The cargo receptor NDP52 initiates selective autophagy by recruiting the ULK complex to cytosol-invading bacteria. Mol. Cell 74, 320-329 e326 (2019).

10. Vargas, J. N. S. et al. Spatiotemporal control of ULK1 activation by NDP52 and TBK1 during selective autophagy. Mol. Cell 74, 347-362 e346 (2019).

11. Johansen, T. \& Lamark, T. Selective autophagy: ATG8 family proteins, LIR motifs and cargo receptors. J. Mol. Biol. 432, 80-103 (2020).

12. Takamura, A. et al. Autophagy-deficient mice develop multiple liver tumors. Genes Dev. 25, 795-800 (2011).

13. Yamasaki, A. et al. Liquidity is a critical determinant for selective autophagy of protein condensates. Mol. Cell 77, 1163-1175 e1169 (2020).

14. Buchan, J. R., Kolaitis, R. M., Taylor, J. P. \& Parker, R. Eukaryotic stress granules are cleared by autophagy and Cdc48/VCP function. Cell 153, 1461-1474 (2013).

15. Zhang, Y. et al. SEPA-1 mediates the specific recognition and degradation of $\mathrm{P}$ granule components by autophagy in C. elegans. Cell 136, 308-321 (2009).

16. Zhang, G., Wang, Z., Du, Z. \& Zhang, H. mTOR regulates phase separation of PGL granules to modulate their autophagic degradation. Cell 174, 1492-1506 e1422 (2018).

17. Sun, D., Wu, R., Zheng, J., Li, P. \& Yu, L. Polyubiquitin chain-induced p62 phase separation drives autophagic cargo segregation. Cell Res. 28, 405-415 (2018).

18. Zaffagnini, G. et al. p62 filaments capture and present ubiquitinated cargos for autophagy. EMBO J. 37, e98308 (2018).

19. Sanchez-Martin, P. et al. NBR1-mediated p62-liquid droplets enhance the Keap1-Nrf2 system. EMBO Rep. 21, e48902 (2020).

20. Turakhiya, A. et al. ZFAND1 recruits $\mathrm{p} 97$ and the $26 \mathrm{~S}$ proteasome to promote the clearance of arsenite-induced stress granules. Mol. Cell 70, 906-919 e907 (2018).

21. Bjorkoy, G. et al. p62/SQSTM1 forms protein aggregates degraded by autophagy and has a protective effect on huntingtin-induced cell death. J. Cell Biol. 171, 603-614 (2005).

22. Inami, Y. et al. Persistent activation of Nrf2 through p62 in hepatocellular carcinoma cells. J. Cell Biol. 193, 275-284 (2011).

23. Kirkin, V. et al. A role for NBR1 in autophagosomal degradation of ubiquitinated substrates. Mol Cell. 33, 505-516. (2009).

24. Matsumoto, G., Wada, K., Okuno, M., Kurosawa, M. \& Nukina, N. Serine 403 phosphorylation of p62/SQSTM1 regulates selective autophagic clearance of ubiquitinated proteins. Mol. Cell 44, 279-289 (2011).

25. Ichimura, Y. et al. Phosphorylation of p62 activates the Keap1-Nrf2 pathway during selective autophagy. Mol. Cell 51, 618-631 (2013).

26. Banani, S. F., Lee, H. O., Hyman, A. A. \& Rosen, M. K. Biomolecular condensates: organizers of cellular biochemistry. Nat. Rev. Mol. Cell Biol. 18, 285-298 (2017).

27. Ribbeck, K. \& Gorlich, D. The permeability barrier of nuclear pore complexes appears to operate via hydrophobic exclusion. EMBO J. 21, 2664-2671 (2002).

28. Lim, J. et al. Proteotoxic stress induces phosphorylation of p62/SQSTM1 by ULK1 to regulate selective autophagic clearance of protein aggregates. PLoS Genet. 11, e1004987 (2015).

29. Jain, A. et al. p62/SQSTM1 is a target gene for transcription factor NRF2 and creates a positive feedback loop by inducing antioxidant response elementdriven gene transcription. J. Biol. Chem. 285, 22576-22591 (2010).

30. Jakobi, A. J. et al. Structural basis of p62/SQSTM1 helical filaments and their role in cellular cargo uptake. Nat. Commun. 11, 440 (2020).

31. Noda, N. N. et al. Structural basis of target recognition by Atg8/LC3 during selective autophagy. Genes Cells 13, 1211-1218 (2008).

32. Wurzer, B. et al. Oligomerization of p62 allows for selection of ubiquitinated cargo and isolation membrane during selective autophagy. elife 4, e08941 (2015).

33. Lee, Y. K. et al. Development of LC3/GABARAP sensors containing a LIR and a hydrophobic domain to monitor autophagy. EMBO J. 36, 1100-1116 (2017).

34. Postic, C. et al. Dual roles for glucokinase in glucose homeostasis as determined by liver and pancreatic beta cell-specific gene knock-outs using Cre recombinase. J. Biol. Chem. 274, 305-315 (1999).

35. Komatsu, M. et al. Homeostatic levels of p62 control cytoplasmic inclusion body formation in autophagy-deficient mice. Cell 131, 1149-1163 (2007).

36. Ichimura, Y. et al. Structural basis for sorting mechanism of p62 in selective autophagy. J. Biol. Chem. 283, 22847-22857 (2008).

37. Eino, A. et al. Sqstm1-GFP knock-in mice reveal dynamic actions of Sqstm1 during autophagy and under stress conditions in living cells. J. Cell Sci. 128, 4453-4461 (2015).

38. Komatsu, M. et al. Impairment of starvation-induced and constitutive autophagy in Atg7-deficient mice. J. Cell Biol. 169, 425-434 (2005).
39. Komatsu, M. et al. The selective autophagy substrate p62 activates the stress responsive transcription factor Nrf2 through inactivation of Keap1. Nat. Cell Biol. 12, 213-223 (2010).

40. Yasuda, D. et al. Synthesis of Keap1-phosphorylated p62 and Keap1-Nrf2 protein-protein interaction inhibitors and their inhibitory activity. Bioorg. Med. Chem. Lett. 26, 5956-5959 (2016).

41. Saito, T. et al. p62/Sqstm1 promotes malignancy of HCV-positive hepatocellular carcinoma through Nrf2-dependent metabolic reprogramming. Nat. Commun. 7, 12030 (2016)

42. Brunger, A. T. Version 1.2 of the crystallography and NMR system. Nat. Protoc. 2, 2728-2733 (2007)

43. Taguchi, K. et al. Keap1 degradation by autophagy for the maintenance of redox homeostasis. Proc. Natl Acad. Sci. USA 109, 13561-13566 (2012).

44. Tsuboyama, K. et al. The ATG conjugation systems are important for degradation of the inner autophagosomal membrane. Science 354, 1036-1041 (2016).

45. Nishida, Y. et al. Discovery of Atg5/Atg7-independent alternative macroautophagy. Nature 461, 654-658 (2009).

46. Hirotsu, Y. et al. Nrf2-MafG heterodimers contribute globally to antioxidant and metabolic networks. Nucleic Acids Res. 40, 10228-10239 (2012).

47. Kobayashi, E. H. et al. Nrf2 suppresses macrophage inflammatory response by blocking proinflammatory cytokine transcription. Nat. Commun. 7, 11624 (2016).

48. Hayashi-Nishino, M. et al. A subdomain of the endoplasmic reticulum forms a cradle for autophagosome formation. Nat. Cell Biol. 11, 1433-1437 (2009).

49. Yla-Anttila, P., Vihinen, H., Jokitalo, E. \& Eskelinen, E. L. 3D tomography reveals connections between the phagophore and endoplasmic reticulum. Autophagy 5, 1180-1185 (2009).

50. Zachari, M. et al. Selective autophagy of mitochondria on a ubiquitinendoplasmic-reticulum platform. Dev. Cell 50, 627-643 e625 (2019).

51. Hollenstein, D. M. \& Kraft, C. Autophagosomes are formed at a distinct cellular structure. Curr. Opin. Cell Biol. 65, 50-57 (2020).

52. Nguyen, T. N. et al. Atg8 family LC3/GABARAP proteins are crucial for autophagosome-lysosome fusion but not autophagosome formation during PINK1/Parkin mitophagy and starvation. J. Cell Biol. 215, 857-874 (2016).

53. Matsumoto, G., Shimogori, T., Hattori, N. \& Nukina, N. TBK1 controls autophagosomal engulfment of polyubiquitinated mitochondria through p62/ SQSTM1 phosphorylation. Hum. Mol. Genet. 24, 4429-4442 (2015).

54. Pilli, M. et al. TBK-1 promotes autophagy-mediated antimicrobial defense by controlling autophagosome maturation. Immunity 37, 223-234 (2012).

55. Mathew, R. et al. Autophagy suppresses tumorigenesis through elimination of p62. Cell 137, 1062-1075 (2009).

56. Ni, H. M. et al. Nrf2 promotes the development of fibrosis and tumorigenesis in mice with defective hepatic autophagy. J. Hepatol. 61, 617-625 (2014).

57. Okawa, H. et al. Hepatocyte-specific deletion of the keap1 gene activates Nrf2 and confers potent resistance against acute drug toxicity. Biochem. Biophys. Res. Commun. 339, 79-88 (2006).

58. Rai, S. et al. The ATG5-binding and coiled coil domains of ATG16L1 maintain autophagy and tissue homeostasis in mice independently of the WD domain required for LC3-associated phagocytosis. Autophagy 15, 599-612 (2019).

59. Gudmundsson, S., Kahlhofer, J., Baylac, N., Kallio, K. \& Eskelinen, E. L. Correlative light and electron microscopy of autophagosomes. Methods Mol. Biol. 1880, 199-209 (2019).

60. Cardona, A. et al. TrakEM2 software for neural circuit reconstruction. PLoS ONE 7, e38011 (2012)

61. Mastronarde, D. N. Automated electron microscope tomography using robust prediction of specimen movements. J. Struct. Biol. 152, 36-51 (2005).

62. Kremer, J. R., Mastronarde, D. N. \& McIntosh, J. R. Computer visualization of three-dimensional image data using IMOD. J. Struct. Biol. 116, 71-76 (1996).

63. Belevich, I., Joensuu, M., Kumar, D., Vihinen, H. \& Jokitalo, E. Microscopy image browser: a platform for segmentation and analysis of multidimensional datasets. PLoS Biol. 14, e1002340 (2016)

64. Kikinis, R., Pieper, S. D. \& Vosburgh, K. In Intraoperative Imaging and ImageGuided Therapy (ed. Jolesz F) (Springer, New York, 2014).

65. Schindelin, J. et al. Fiji: an open-source platform for biological-image analysis. Nat. Methods 9, 676-682 (2012)

66. Arai, R. \& Waguri, S. Improved electron microscopy fixation methods for tracking autophagy-associated membranes in cultured mammalian cells. Methods Mol. Biol. 1880, 211-221 (2019).

67. Waguri, S. \& Komatsu, M. Biochemical and morphological detection of inclusion bodies in autophagy-deficient mice. Methods Enzymol. 453, 181-196 (2009).

68. Storey, J. D. A direct approach to false discovery rates. J. R. Stat. Soc. B 64, 479-498 (2002).

69. Storey, J. D., Bass, A. J., Dabney, A. \& Robinson, D. qvalue: Q-value estimation for false discovery rate control. $R$ package version 2.18.0, Bioconductor qualue. http://github.com/jdstorey/qvalue. (2019). 
70. Huang da, W., Sherman, B. T. \& Lempicki, R. A. Systematic and integrative analysis of large gene lists using DAVID bioinformatics resources. Nat. Protoc. 4, 44-57 (2009).

71. Hubstenberger, A. et al. P-body purification reveals the condensation of repressed mRNA regulons. Mol. Cell 68, 144-157 e145 (2017).

\section{Acknowledgements}

We thank all members of Komatsu's lab. and Sánchez-Martín Pablo for their helpful comments and discussion. S. Kageyama is supported by a Grant-in-Aid for Scientific Research (C) (20K06549). Y.-S.S. is supported by a Grant-in-Aid for Scientific Research (C) (19K15043). D.N. is supported by a Grant-in-Aid for Early-Career Scientists (19K16344). N.N.N. is supported by a Grant-in-Aid for Scientific Research on Innovative Areas (19H05707). M.K. is supported by a Grant-in-Aid for Scientific Research on Innovative Areas (19H05706), a Grant-in-Aid for Scientific Research (B) (18H02611), the Japan Society for the Promotion of Science (an A3 foresight program), and the Takeda Science Foundation (to M.K.). This work was supported by JSPS KAKENHI Grant Number JP 16H06276 (AdAMS). E.L.E. and S.R.G. were supported by the Academy of Finland and Magnus Ehrnrooth Foundation. The Electron Microscopy Laboratory at Institute of Biomedicine, University of Turku, in thanked for technical help and availability of instruments.

\section{Author contributions}

M.K. designed and directed the study. E-L.E., S.W., N.N.N., and M.K. wrote the manuscript. S. Kageyama, Y-S.S., Y.I., and T.U. performed the cell biological experiments. S.R.G. and E-L.E. carried out CLEM and tomography. N.T. and S.W. performed histological and microscopic analyses with genetic modified mice. D.N. and N.N.N. performed GUV experiments. M.A. and K.S. generated genetic modified mice. S. Kazuno and Y.M. conducted proteomic analyses with genetically modified mice. H.M. searched Nrf2-targets among upregulated proteins in genetically modified mice. J-A.L. provided HyD-LIR probe. T.O. developed and provided KMN003. S.O. and N.O. conducted statistic analysis of proteomics data. All authors discussed the results and commented on the manuscript.

\section{Competing interests}

The authors declare no competing interests.

\section{Additional information}

Supplementary information is available for this paper at https://doi.org/10.1038/s41467020-20185-1.

Correspondence and requests for materials should be addressed to E.-L.E. or M.K.

Peer review information Nature Communications thanks Sihem Boudina, Muriel Mari, $\mathrm{Li} \mathrm{Yu}$, and other, anonymous reviewers for their contributions to the peer review of this work. Peer review reports are available.

Reprints and permission information is available at http://www.nature.com/reprints

Publisher's note Springer Nature remains neutral with regard to jurisdictional claims in published maps and institutional affiliations.

Open Access This article is licensed under a Creative Commons Attribution 4.0 International License, which permits use, sharing, adaptation, distribution and reproduction in any medium or format, as long as you give appropriate credit to the original author(s) and the source, provide a link to the Creative Commons license, and indicate if changes were made. The images or other third party material in this article are included in the article's Creative Commons license, unless indicated otherwise in a credit line to the material. If material is not included in the article's Creative Commons license and your intended use is not permitted by statutory regulation or exceeds the permitted use, you will need to obtain permission directly from the copyright holder. To view a copy of this license, visit http://creativecommons.org/ licenses/by/4.0\%

(C) The Author(s) 2021 